SANDIA REPORT

SAND2006-3517

Unlimited Release

Printed June 2006

\title{
EM Threat Analysis for Wireless Systems
}

R. J. Burkholder, I. J. Gupta, and P. Schniter

The Ohio State University ElectroScience Laboratory

Department of Electrical Engineering

R. J. Mariano, Multi-Physics Modeling \& Simulation Department

Prepared by

Sandia National Laboratories

Albuquerque, New Mexico 87185 and Livermore, California 94550

Sandia is a multiprogram laboratory operated by Sandia Corporation,

a Lockheed Martin Company, for the United States Department of Energy's

National Nuclear Security Administration under Contract DE-AC04-94-AL85000.

Approved for public release; further dissemination unlimited.

国 Sandia National Laboratories 
Issued by Sandia National Laboratories, operated for the United States Department of Energy by Sandia Corporation.

NOTICE: This report was prepared as an account of work sponsored by an agency of the United States Government. Neither the United States Government, nor any agency thereof, nor any of their employees, nor any of their contractors, subcontractors, or their employees, make any warranty, express or implied, or assume any legal liability or responsibility for the accuracy, completeness, or usefulness of any information, apparatus, product, or process disclosed, or represent that its use would not infringe privately owned rights. Reference herein to any specific commercial product, process, or service by trade name, trademark, manufacturer, or otherwise, does not necessarily constitute or imply its endorsement, recommendation, or favoring by the United States Government, any agency thereof, or any of their contractors or subcontractors. The views and opinions expressed herein do not necessarily state or reflect those of the United States Government, any agency thereof, or any of their contractors.

Printed in the United States of America. This report has been reproduced directly from the best available copy.

Available to DOE and DOE contractors from

U.S. Department of Energy

Office of Scientific and Technical Information

P.O. Box 62

Oak Ridge, TN 37831

Telephone: $\quad$ (865) 576-8401

Facsimile: $\quad$ (865) 576-5728

E-Mail: $\quad$ reports@adonis.osti.gov

Online ordering: http://www.doe.gov/bridge

Available to the public from

U.S. Department of Commerce

National Technical Information Service

5285 Port Royal Rd

Springfield, VA 22161

Telephone: $\quad$ (800) 553-6847

Facsimile: $\quad$ (703) 605-6900

E-Mail: $\quad$ orders $@$ ntis.fedworld.gov

Online order: $\quad$ http://www.ntis.gov/help/ordermethods.asp?loc=7-4-0\#online 
SAND2006-3517

Unlimited Release

Printed June 2006

\title{
EM Threat Analysis for Wireless Systems
}

\author{
R.J. Burkholder, I.J. Gupta, and P. Schniter \\ The Ohio State University ElectroScience Laboratory \\ Department of Electrical Engineering \\ R. J. Mariano ${ }^{1}$ \\ Multi-Physics Modeling \& Simulation Department \\ Sandia National Laboratories \\ Livermore, California 94551-0969
}

\begin{abstract}
Modern digital radio systems are complex and must be carefully designed, especially when expected to operate in harsh propagation environments. The ability to accurately predict the effects of propagation on wireless radio performance could lead to more efficient radio designs as well as the ability to perform vulnerability analyses before and after system deployment.

In this report, the authors - experts in electromagnetic (EM) modeling and wireless communication theory - describe the construction of a simulation environment that is capable of quantifying the effects of wireless propagation on the performance of digital communication.
\end{abstract}

\footnotetext{
${ }^{1}$ This report was written by the Ohio State University authors under contract with Sandia National Laboratories, Livermore, CA, under PO 450880. Robert Mariano was the contractor overseer.
} 


\section{Acknowledgements}

The authors would like to acknowledge J. Brian Grant, from ANT-S, Livermore, CA, for his technical expertise during the development of this report. 


\section{Contents}

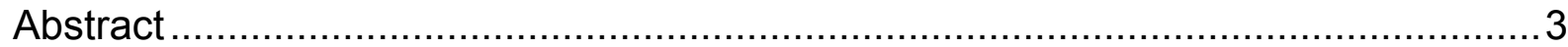

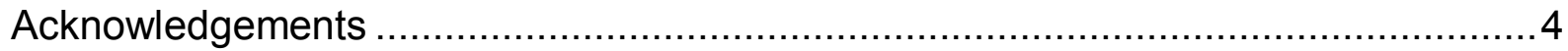

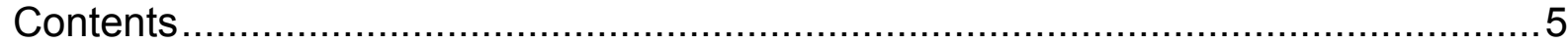

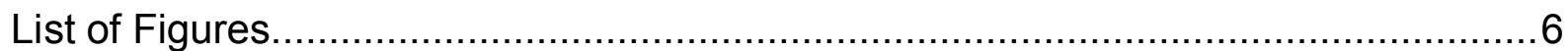

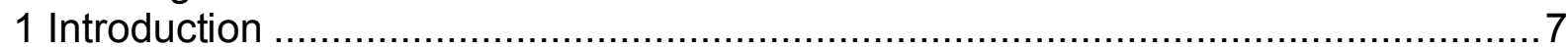

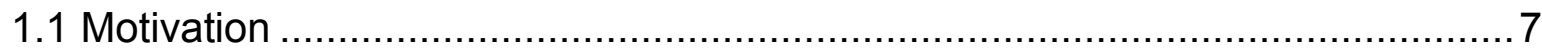

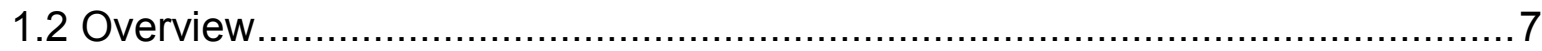

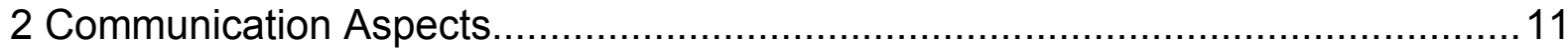

2.1 The Basics of Wireless Digital Communication............................................11

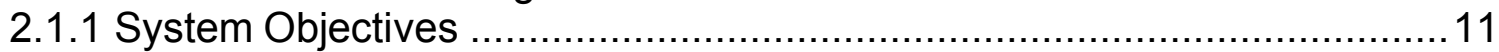

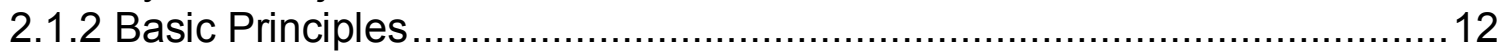

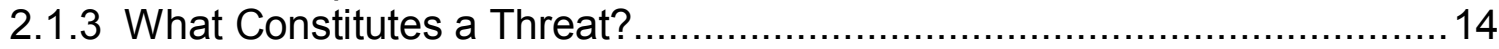

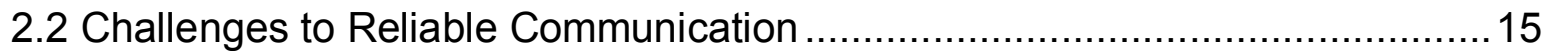

2.2.1 The Multipath Fading Phenomenon ……........................................ 15

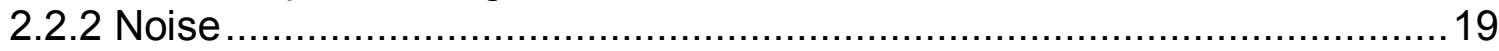

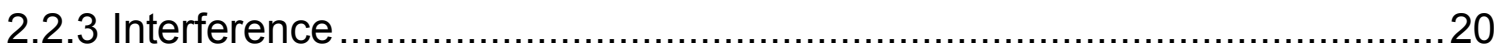

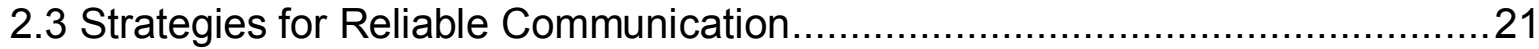

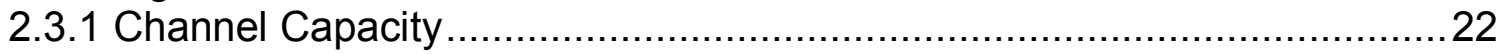

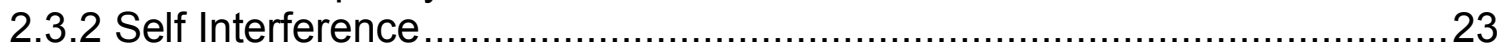

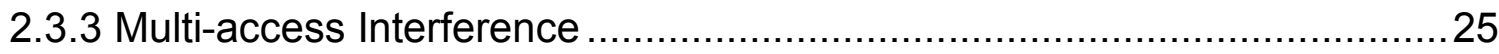

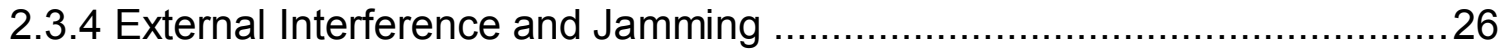

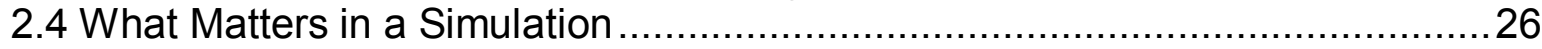

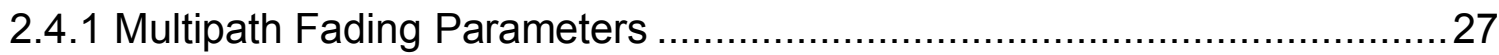

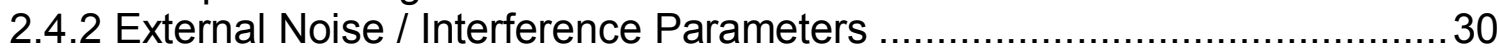

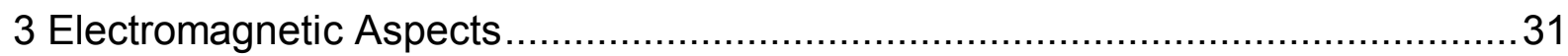

3.1 The Basic EM Components of Wireless Transmission ...................................

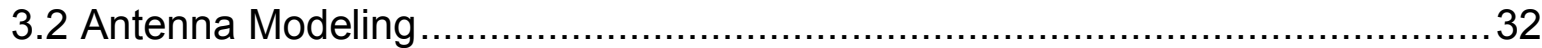

3.2.1 Conventional Analysis Methods for Antennas ...................................... 32

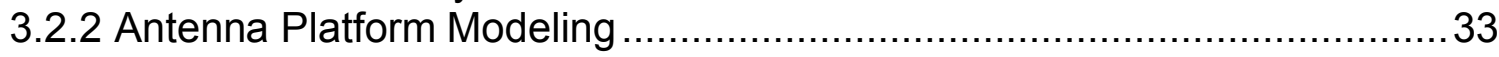

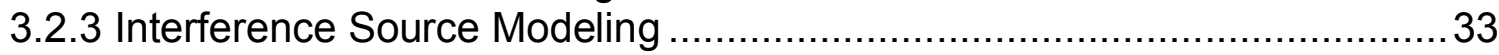

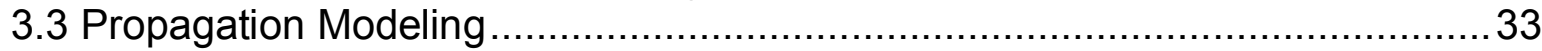

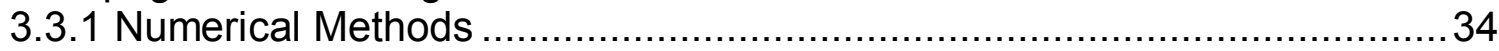

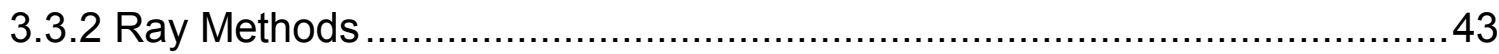

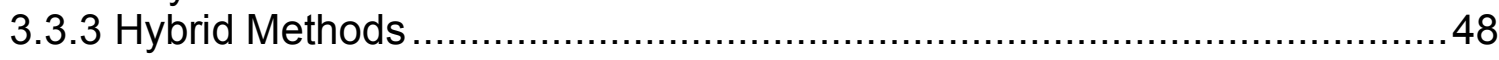

3.3.4 Summary of Modeling Methods and Applications ................................51

3.4 Recommended Approaches to Site-Specific Modeling …..............................53

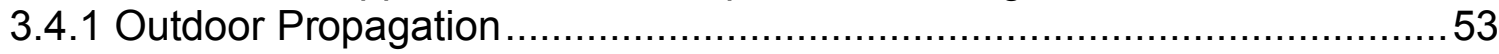

3.4.2 Outdoor-to-Indoor Propagation, Large Room......................................53

3.4.3 Outdoor-to-Indoor Propagation, Small Room or Enclosure ......................53

3.4.4 Indoor Propagation, Large Room or Buiolding …................................54

3.4.5 Indoor Propagation, Small Room or Enclosure ...................................54

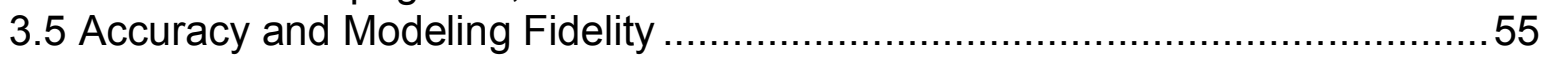

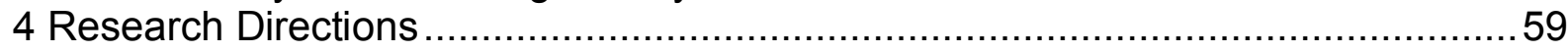

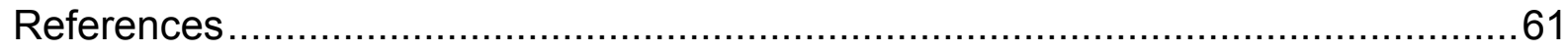




\section{List of Figures}

Figure 1: The proposed two-step approach: statistical performance characterization via empirical averaging of deterministic simulations.................................. 9

Figure 2: Effect of noise and distortion on single-bit communication. Here the received waveform looks more like the false distorted hypothesis than the true one, and so an error occurs. ........................................................... 13

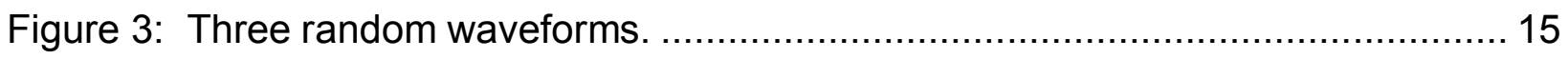

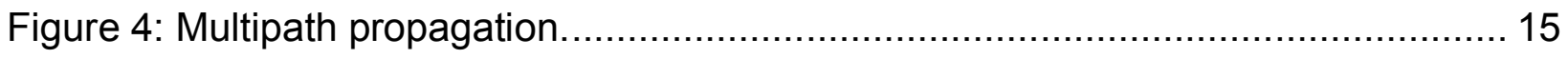

Figure 5: A "ring of scatterers" with equal propagation delays .................................. 16

Figure 6: BER of binary phase shift keying over a flat Rayleigh-fading channel in the

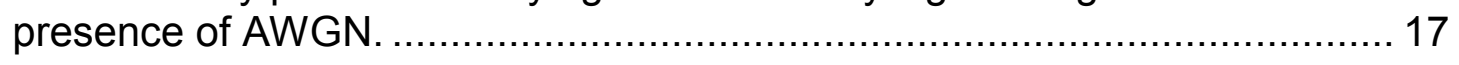

Figure 7: Time-varying frequency response due to multipath fading.......................... 18

Figure 8: BER of binary phase shift keying over $D$ flat Rayleigh diversity branches in the presence of AWGN.

Figure 9: (a) Maximum spectral efficiency (bits/s/Hz) of the AWGN channel versus $S N R=\frac{P}{W N_{0}} . \quad$ (b) Capacity (Mbits/s) versus bandwidth $W(\mathrm{MHz})$ when $\frac{P}{N_{0}}=10^{6}$. The dashed line indicates limit as $W \rightarrow \infty \ldots \ldots \ldots \ldots \ldots \ldots \ldots \ldots . . .23$

Figure 10: Graphical representation of various modulation schemes. Each colored block represents that time-frequency support of a different symbol. ............. 23

Figure 11: Graphical representations of various multi-access schemes. Each colored block represents the time-frequency support of a different user.

Figure 12: Mean signal power as a function of distance between transmitter and receiver under different amounts of local spatial averaging. (Redrawn from

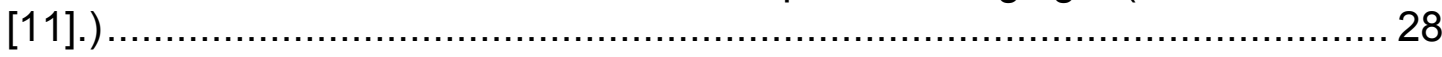

Figure 13: Delay power profiles: (a) exponential, (b) uniform.................................... 29

Figure 14: Doppler power profiles: (a) bathtub, (b) uniform.................................... 29

Figure 15: Basic components of a wireless communications system pertinent to EM

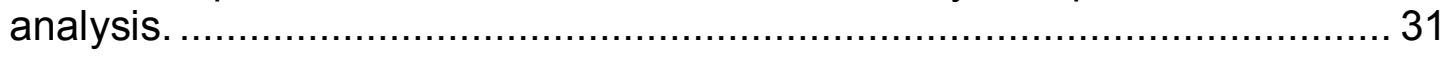

Figure 16: FDTD problem domains with a structured mesh. .................................... 37

Figure 17: Unstructured FEM mesh in an enclosed volume.................................... 39

Figure 18: Equivalent source problem for method of moments. ............................... 42

Figure 19: Ray tracking methods for propagation modeling …................................ 45

Figure 20: Outdoor propagation problems using ray-tracing. ................................ 47

Figure 21: Domain decomposition of a wireless propagation problem for hybrid analysis. 


\section{Introduction}

\subsection{Motivation}

Modern digital radio systems are complex and must be carefully designed, especially when expected to operate in harsh propagation environments. The ability to accurately predict the effects of propagation on wireless radio performance could lead to more efficient radio designs as well as the ability to perform vulnerability analyses before and after system deployment.

In this report, the authors - experts in electromagnetic (EM) modeling and wireless communication theory - describe the construction of a simulation environment that is capable of quantifying the effects of wireless propagation on the performance of digital communication.

\subsection{Overview}

The complexity and variability of EM propagation, as well as the complexity of the radios themselves, make it challenging to accurately characterize/predict the performance of radio systems. Consider that, every minute, a modern digital wireless system transmits and receives millions of data packets, each affected by a different set of propagation parameters. The parameters change as a result of centimeter-scale changes in the physical geometry between the transmitter, receiver, and nearby scattering objects, as well as by changes in the interference patterns contributed by external sources of EM radiation. The key to simulating these complex systems is to

a) describe the infinitely complex EM propagation/interference environment by a small subset of essential parameters, and

b) accurately model these essential parameters for a given propagation/interference environment.

One could go further and include the "meta task"

c) identify the subset of important propagation environments to characterize,

but this will not be the focus of this report. Corresponding to the first two points above, Section 2 explains which propagation/interference parameters are essential (by reviewing the operation of modern digital wireless radios), and Section 3 describes how these parameters can be accurately modeled (by reviewing the state-of-the-art in EM simulation).

Throughout, we advocate a statistical approach to performance characterization. This approach is motivated by the high variability of the propagation environment and the inherent averaging that occurs when communicating many bits. For example, when considering propagation environments that impart spectral nulls on the received spectrum, the exact location of every null at every time instant is not critical to predicting the average bit error rate (BER) of the system; rather, the average spacing between (or expected number of ) nulls might be sufficient. If, 
instead, we were interested in ascertaining the success probability for communication of a single packet at a particular time instant in a very particular environment (i.e., where the physical geometry and interference spectrum are known perfectly), then exact null locations would be much more important. This latter problem, however, is neither typical nor practical due to the accuracy required in environmental specification.

The sufficiency of statistical modeling is fortunate in the sense that it dramatically reduces the required number of simulation parameters, although it does not necessarily reduce the required accuracy of modeling. This is a consequence of the fact that communication systems can be very sensitive to the values of particular propagation/interference parameters. For example, "threshold effects", where robust operation is maintained throughout a particular operating range, but catastrophic operation occurs beyond, are not unusual. Furthermore, communication system performance is often sensitive to system design/implementation details, making it impossible to precisely quantify the BER of a generic wireless radio.

While we are interested in a statistical characterization of the propagation/interference environment, the vast majority of EM simulations are conducted in a deterministic framework. For example, it is typical for an EM simulation to produce a model of the impulse response for a particular physical geometry. The performance characterization of digital radios is typically conducted in a deterministic framework as well, due to the complexity of the radio system and the sensitivity to its design details. For example, it is typical to quantify the BER of a data packet given a particular impulse response and particular data, noise, and interference realizations. To bridge the gap between the statistical and deterministic worlds, we propose a two-step approach to performance characterization, described below and illustrated in Figure 1. We will henceforth use the term "channel" to describe the combined propagation/interference characteristics.

1.

a) For a particular environmental specification (e.g., an office), generate several sets of physical specifications.

b) For each set of physical specifications (e.g., room geometry and locations of objects in room), characterize the essential deterministic channel parameters via numerical EM simulation.

c) Given several sets of deterministic channel parameters (e.g., impulse response), empirically estimate the essential statistical channel parameters.

2.

a) Given a set of statistical channel parameters (e.g., power delay profile, interference spectrum), generate several sets of deterministic channel/signal realizations (e.g., impulse response, bit sequence, noise waveform).

b) For each channel/signal realization, numerically simulate the communication system and generate deterministic performance parameters (e.g., the number of bit errors).

c) From the deterministic performance parameters, empirically estimate the statistical performance parameters (e.g., average BER). 


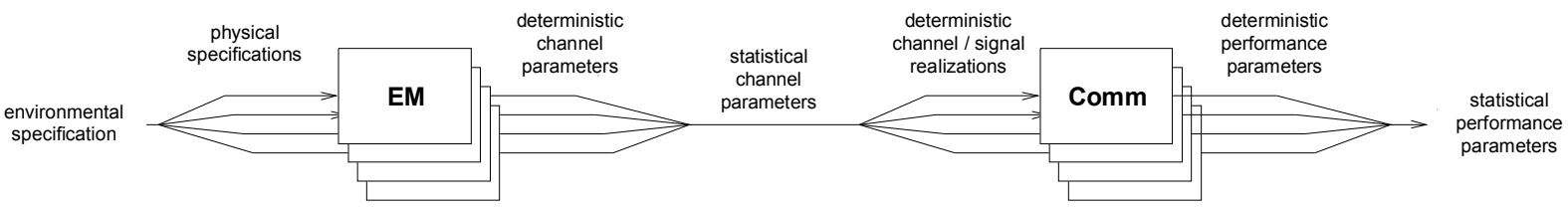

Figure 1: The proposed two-step approach: statistical performance characterization via empirical averaging of deterministic simulations.

The required number of EM simulations is expected to be fewer than the required number of communication simulations by several orders of magnitude. This is because characterization of a communication system generating BERs on the order of, say, $1 \times 10^{-6}$ requires several million bits, whereas only dozens of EM simulations should suffice to characterize statistical channel parameters for a given environmental specification.

Among the essential channel statistics are some that quantify the rate of channel variation. It is possible to estimate these parameters from deterministic (fixed) EM simulations by scheduling (i.e., "stepping") a sequence of physical geometries in accordance with prototypical geometric trajectories. For example, if we are interested in an environmental specification where the receiver moves at a particular velocity, we could generate a sequence of physical geometries which samples a typical receiver trajectory. 
Intentionally Left Blank 


\section{Communication Aspects}

This section addresses the question: "Which channel parameters should be measured/modeled in the testing/simulation of a wireless digital communication system?". To answer this question, we must first understand the mechanisms by which transmission errors occur, which in turn requires an understanding of how information is transmitted and what happens to this information on its way to the receiver.

\subsection{The Basics of Wireless Digital Communication}

In Section 2.1 we discuss the design and operation of wireless digital communication systems from a high level, laying the groundwork for more in-depth discussions of salient channel/interference effects which will eventually guide the construction of simulation/test strategies.

\subsubsection{System Objectives}

It is important to understand that digital communication systems come in many flavors; channel/interference qualities that significantly impact the operation of one system may be inconsequential to another, and vice-versa. Associated with every communication system is a particular set of design objectives, and these objectives help define the behavior of a given system under both typical and atypical operating conditions. For example, application-level objectives might include constraints on minimum data rate, maximum bit error rate, and maximum delay; system-level objectives might include constraints on occupied bandwidth, radiated power, and robustness to co-channel interference; terminal-level objectives might include constraints on size, weight, average power consumption, peak power consumption, complexity, and cost. In secure applications, the objectives might include minimizing the probabilities of detection and intercept.

Implicit in the design of any system are assumptions on the "typical" operating conditions. Under typical operating conditions, any well-designed system will satisfy it's design objectives (e.g., operate at a very low BER). Under atypical operating conditions, however, even welldesigned systems may fail to meet their objectives. As an example, consider that off-the-shelf North American digital terrestrial television receivers are likely to fail if used at highway speeds, or even when used at a fixed location near a busy high way, due to a complicated interference pattern that changes too quickly for the receiver to keep up with. In contrast, cellular phones are quite reliable in these environments, though they too have their limitations (e.g., tunnels and elevators). As another example, locally jamming a cellular communication system is somewhat easy to do, although jamming a spread-spectrum military communication system is - by design much more difficult. It is not that the militarily communication system is necessarily more sophisticated than the consumer system; rather, the military system has been designed to tolerate a much higher level of interference (usually at the expense of some other feature like bandwidth efficiency). To understand how a particular channel/interference artifact will impact a particular system requires some understanding of the basic principles of digital communication. 


\subsubsection{Basic Principles}

In digital communication, we assume that the information is represented by a sequence of bits. Rather than communicating these bits one at a time, we typically collect groups of bits into "symbols" and transmit these symbols one at time. To do this, we associate each symbol with a unique analog waveform, and transmit this waveform through the wireless medium. For example, if there are 4 bits (i.e., 16 possibilities) per symbol, then we might construct a set of 16 waveforms and transmit one of these 16 waveforms during each symbol interval.

Systems that employ error control coding work according to the same principle, though a larger number of bits are collected to form a "codeword" which is associated with a sequence of symbols and, hence, a unique analog waveform. By making only a fraction of the symbol sequences "valid," a controlled redundancy can be incorporated which significantly increases the system's robustness to error. For example, say we have 32 bits to communicate and, as before, we use symbols from an "16-ary" alphabet (i.e., an alphabet of size 16). An uncoded system would split these 32 bits into a length- 8 symbol sequence (i.e., allocating 4 information bits to each symbol), while a "rate-1/3" coded system would code these 32 bits into a length-24 symbol sequence (i.e., allocating $4 / 3$ information bits to each symbol on average). In the uncoded system, every one of the $2^{32}=16^{8}$ possible symbol sequences would be valid, while in the coded system, only $2^{32}$ out of $16^{24}$ possible symbol sequences would be valid. By carefully choosing the subset of valid sequences, a good code can greatly increase the robustness to transmission errors.

The receiver observes a corrupted version of the transmitted waveform, from which it tries to infer which symbol sequence was most likely transmitted. Notice that the corrupted received waveform contains two components: a distorted version of the transmitted waveform plus a noise/interference waveform that has nothing to do with the transmitted waveform. Assume, for the moment, that the receiver is somehow able to perfectly learn the distortion mechanism and that no two distorted hypotheses look identical. Then, in the absence of noise/interference, the receiver would never make a decision error! In practice, however, some noise/interference is always present, and decision errors will occur when the noise/interference causes the received signal to look more like an incorrect distorted hypothesis than the true distorted waveform. Clearly, then, the success of this scheme depends on how "different" the distorted hypotheses look, or, more specifically, how significant the noise/interference is relative to these differences. Figure 2 gives an illustration of the process for the simple case of singlebit communication. 


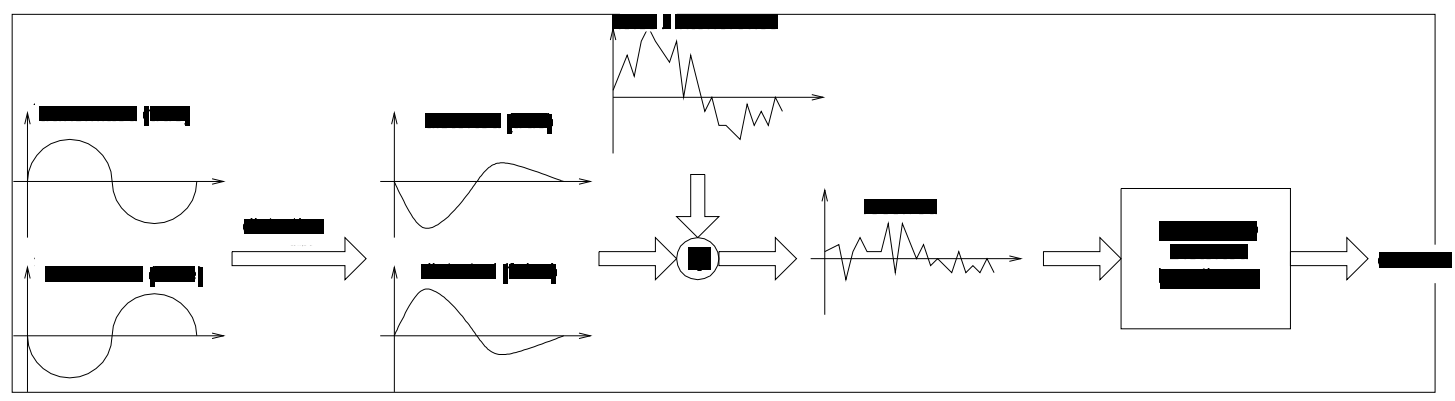

Figure 2: Effect of noise and distortion on single-bit communication. Here the received waveform looks more like the false distorted hypothesis than the true one, and so an error occurs.

In practice, learning the distortion mechanism and compensating for it can be difficult and computationally intensive. This motivates designers to choose a transmission scheme whose waveforms are robust to the channel's distortion processes. Take, for example, a channel with a $1 \mu \mathrm{s}$ echo decay. If the transmitted waveform's duration is $10 \mu \mathrm{s}$, then the echoes will only cause inter-symbol interference to $10 \%$ of the waveform, and decent symbol estimates should be obtainable without a detailed knowledge of the echo pattern. On the other hand, if the transmitted waveform's duration is $0.1 \mu \mathrm{s}$, then the echoes will cause interference among 10 neighboring symbols. Resolving this inter-symbol interference (ISI) requires an accurate knowledge of the echo pattern's details.

Even with robust transmission waveforms, there generally remains some need to learn/track at least some of the channel's features. In practice, these channel estimates are never perfectly accurate, and the effects of this inaccuracy lead to various forms of residual "self-interference" (e.g., residual ISI in the example above). This residual self-interference combines with the external noise/interference to cause symbol estimation errors.

To summarize, a group of bits determines a sequence of symbols, and the symbol sequence specifies which waveform is transmitted across a communications channel. The channel distorts this waveform and adds external noise/interference. After obtaining an estimate of the distortion mechanism, the receiver can build a set of (distorted) hypotheses. To infer which symbol sequence was sent, it compares the noisy received waveform to these hypotheses and looks for the best "match." An error occurs when the external noise/interference plus the residual selfinterference combine to confuse the receiver into incorrectly inferring the correct hypothesis. These errors are more likely to occur when the noise/interference is significant with respect to the differences between hypotheses. When channel distortion causes any two hypotheses to look very similar, it will be easy for the noise/distortion to induce decision errors. In subsequent sections, we examine waveform designs, channel distortion mechanisms, noise/interference characteristics, and their interactions in more detail. 


\subsubsection{What Constitutes a Threat?}

It is important to keep in mind that any reasonable communication system is designed to provide acceptable performance under expected channel/interference conditions, whether or not these expected conditions are "severe" or not. Unacceptable performance will occur only when there is a mismatch between the expected and actual channel/interference conditions, and only when the system is sensitive to this mismatch.

To predict whether or not a system will fail requires that we know the set of channel parameters to which the transmission/reception strategy is sensitive, as well as the values of those parameters. Furthermore, we require that the parameterization be reasonably simple. For example, it would not be practical to describe the noise process by an infinitely complicated amplitude waveform that varies over space and time. Rather, we are inclined to model the noise as a Gaussian random process parameterized by mean and variance, and then analyze the probability that the noise causes a bit error. Similarly, the infinitely complicated nonlinear channel distortion mechanism might be modeled as a linear system and parameterized by a timevariant impulse (or frequency) response. Or, if further simplification is needed, the impulse response coefficients could be modeled as zero-mean Gaussian random variables with a particular correlation. The statistical channel parameterization could be used to analyze the average probability of a bit error, where the averaging is performed over channel realizations.

Generally, a very detailed knowledge of the transmission and reception schemes is required to ascertain the effect of channel parameters on performance criteria like average bit error rate. In addition to knowing the modulation and coding schemes employed by the system, it is important to know - in reasonable detail - the algorithms (e.g., timing recovery, channel estimation, equalization, decoding) used by the receiver. In fact, because the bit error rate is a very complicated function of the channel parameters, it is usually numerically evaluated through computer simulations with randomly generated channel and noise realizations.

Note that, when predicting average system behavior, it is sufficient to know only the average channel behavior. However, caution must be employed in ascertaining exactly which "average" channel parameters matter. For example, Figure 3 shows three random waveforms with identical mean and variance. These waveforms might represent, e.g., noise waveforms or the channel gain over time. While the autocorrelation properties (i.e., rate of variation) of waveforms (a) and (b) are similar, that of waveform (c) differs. Consequently, though waveforms (a) and (b) are far from identical, they might yield similar average performance. In contrast, waveform (c) might yield a very different average performance (e.g., it's future values are easier to predict given past samples). 


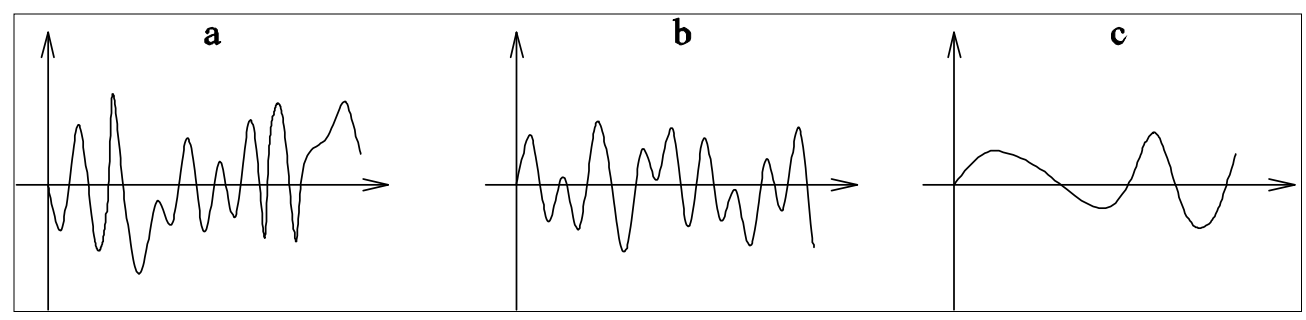

Figure 3: Three random waveforms.

\subsection{Challenges to Reliable Communication}

Section 2.1 discussed the high-level interplay between modulation, channel distortion, noise/interference, and demodulation. Here, in Section 2.2, we take a more detailed look at the channel and interference characteristics that are likely to affect wireless communication systems. Later, in Section 2.3, we discuss how specific communication schemes will be affected by specific channel/interference characteristics.

\subsubsection{The Multipath Fading Phenomenon}

Multipath fading is perhaps the most significant distortion mechanism characterizing the wireless communication channel. "Multipath" refers to the tendency for the signal to propagate along several paths between the transmitter and receiver (see Figure 4). While a direct path may exist in some line-of-site applications, most paths are reflected or "scattered" by foreign objects. Figure 5 illustrates the "ring of scatterers" concept, which says that single reflections from any scatterers on an ellipse (whose foci are defined by the transmitter and receiver locations) yield propagation paths of identical delay. The superposition of identically delayed paths - each with a possibly unique amplitude and phase - can boost or attenuate the amplitude of the signal component received at that particular delay. We say that a signal component fades when its amplitude becomes small relative to the noise.

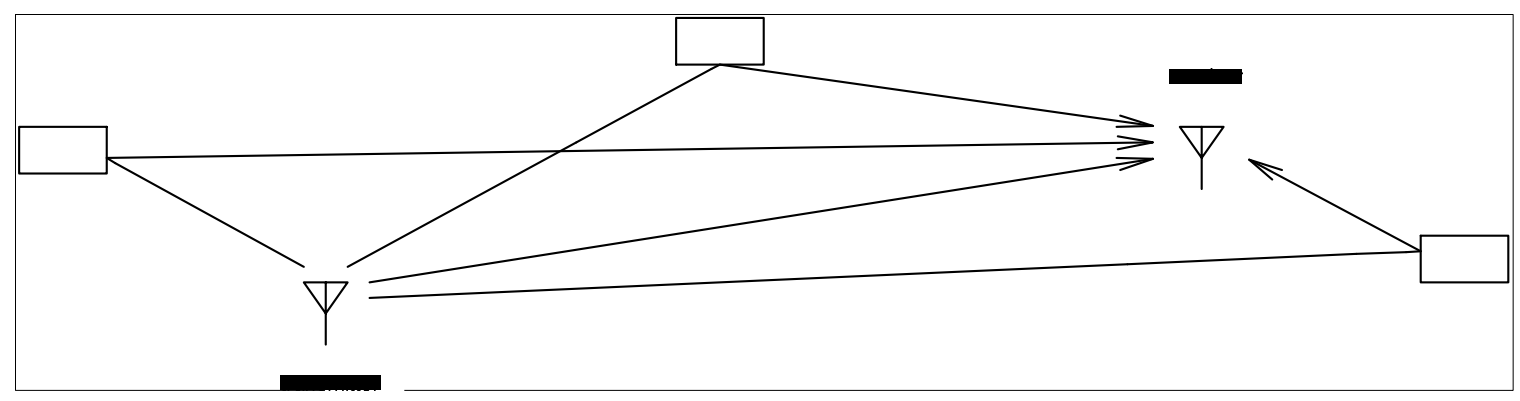

Figure 4: Multipath propagation. 


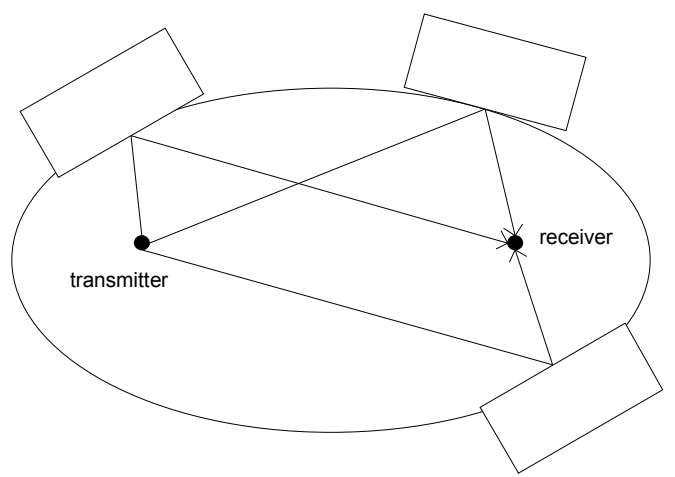

Figure 5: A "ring of scatterers" with equal propagation delays

With many reflectors and no ${ }^{2}$ line-of-sight path, the superposition of paths results in channel gains that can be well modeled by zero-mean complex Gaussian random variables. In this case, the channel is referred to as "Rayleigh fading." With Rayleigh fading, the probability that the signal is overwhelmed by additive white Gaussian noise (AWGN) turns out to be proportional ${ }^{3}$ to 1/SNR, where SNR denotes the ratio of average received signal energy to average received noise energy per symbol [1, p. 55]. In contrast, in the absence of fading, the probability that the signal becomes overwhelmed by AWGN is proportional to $1=\exp (\mathrm{SNR})$. Figure 6 illustrates the effect of Rayleigh fading on a simple binary communication system in AWGN. Note how significant the effects of fading are at medium and high SNR.

When the multipath delay spread is small relative to a symbol interval, the fading is known as "flat" because it affects all frequency components in the transmitted signal identically. When the multipath delay spread is large relative to a symbol interval, however, the fading becomes frequency-selective (FS), meaning that some frequency ranges within the transmitted bandwidth fade independently of others. When there exists relative motion between the transmitter, receiver, and scattering objects, the gain due to fading changes over time, giving rise to timeselective (TS) fading. The amount of motion needed to effect a significant change is on the order of a quarter wavelength. Figure 7 shows a prototypical time-varying frequency response of a channel that exhibits both TS and FS fading. When multiple or directional antennas are used, the fading is said to be spatially selective (SS). The fading gains observed at two antennas are said to behave independently when the antennas are separated by a quarter wavelength or more. To quantify the sensitivity of TS and SS fading, we note that, at $3 \mathrm{GHz}$, a quarter wavelength is 2.5 centimeters, and, at higher frequencies, a quarter wavelength is even smaller.

\footnotetext{
${ }^{2}$ With a line-of-sight path, the superposition results in channel gains that can be well modeled by a non-zero-mean complex Gaussian random variables. In this case, the channel is referred to as "Ricean fading."

${ }^{3}$ This approximation gets more accurate at high SNR.
} 


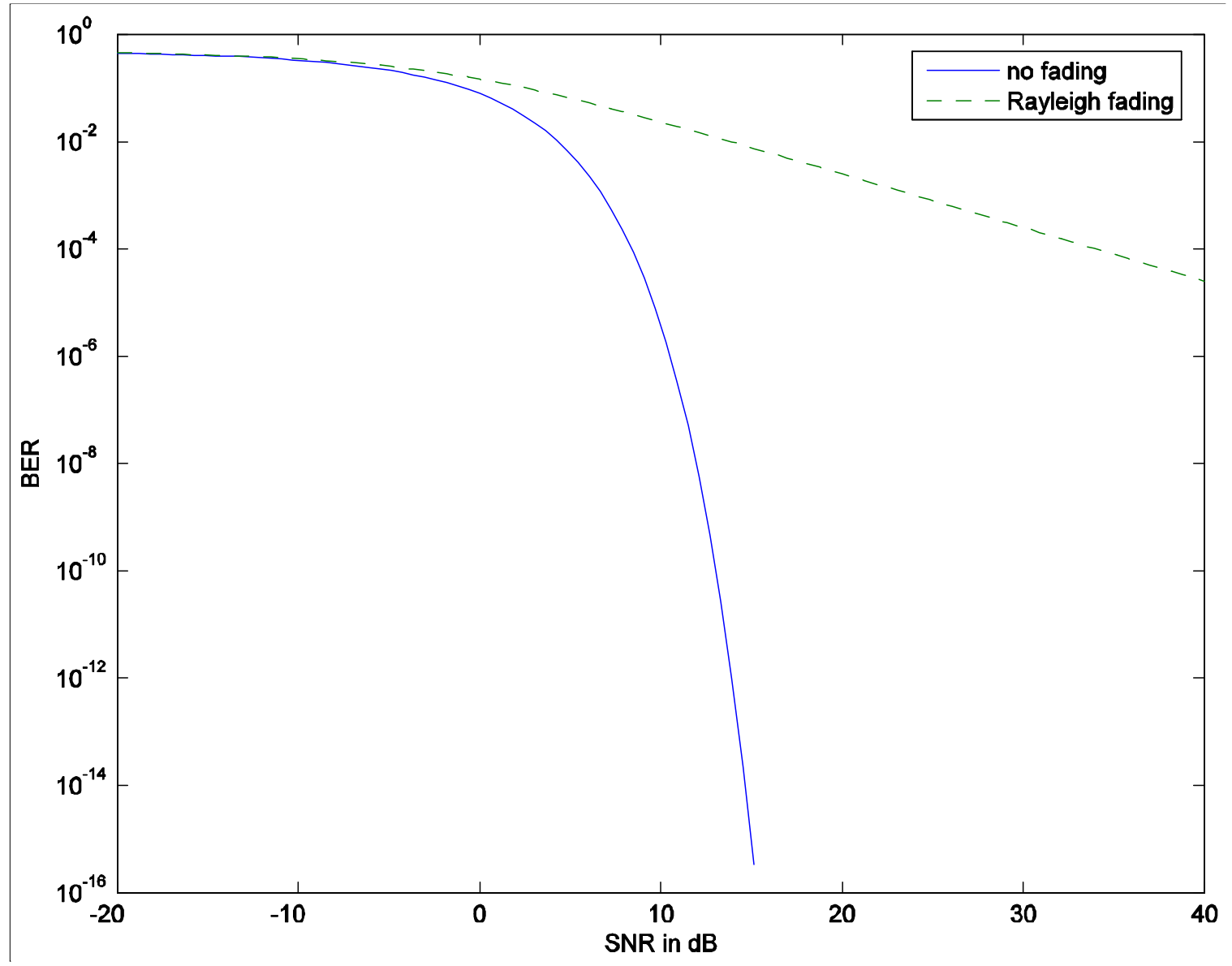

Figure 6: BER of binary phase shift keying over a flat Rayleigh-fading channel in the presence of AWGN. 


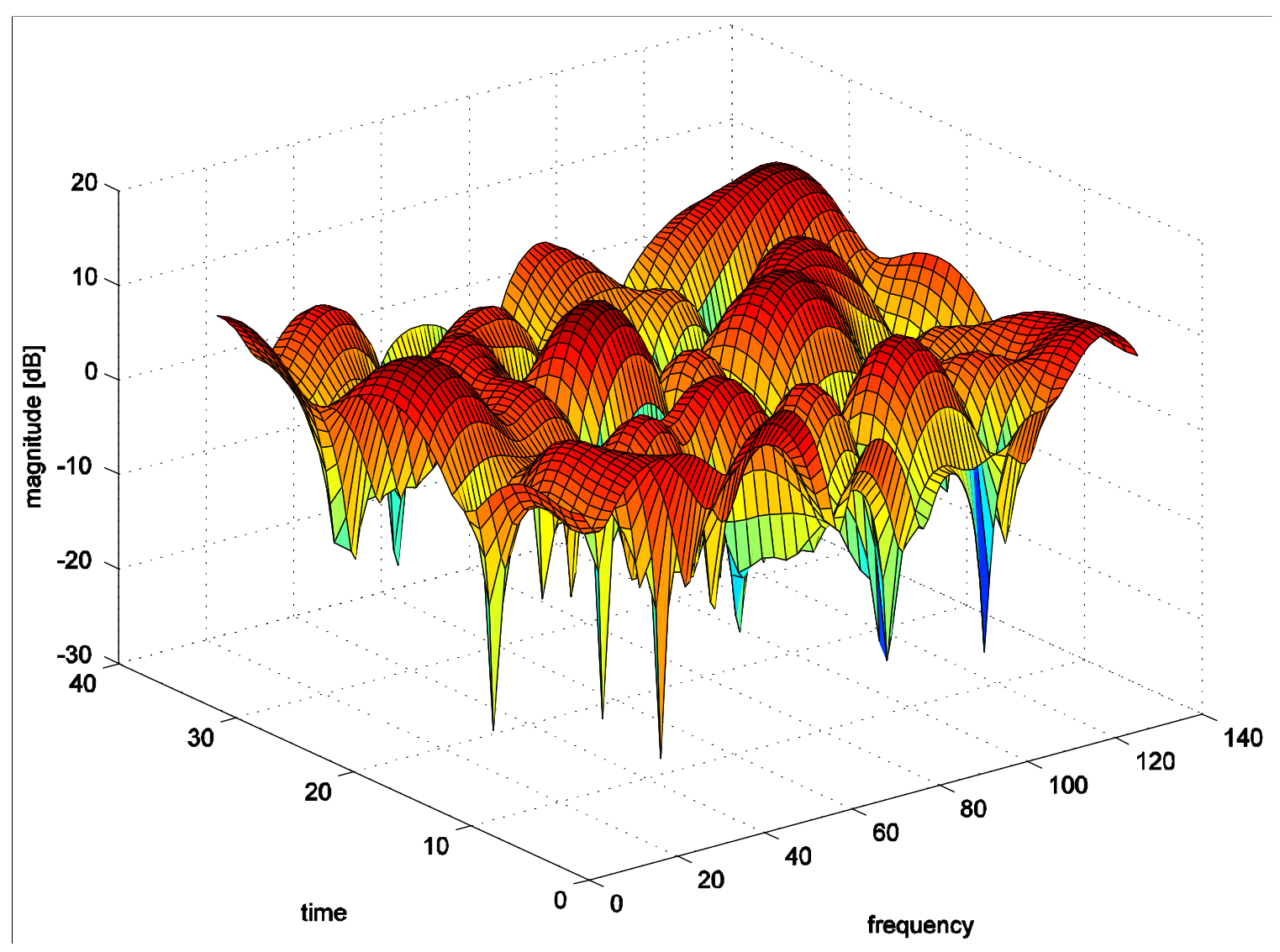

Figure 7: Time-varying frequency response due to multipath fading.

Multipath propagation requires careful mitigation at the receiver. Recall that multi path fading causes the received signal to fade at particular times, frequencies, and locations. If the signal from an information bit is received at only one time/frequency/location, then there will be no way of reliably recovering this bit when that time/frequency/location fades. If, on the other hand, the signal from this information bit is received at multiple times/frequencies/locations, then it should be possible to reliably infer the bit unless every one of these times/frequencies/locations has faded simultaneously - a situation which occurs very rarely. When bits are transmitted and received over D independently fading time/frequency/location coordinates, we say that we achieve $\mathrm{D}^{\text {th }}$-order diversity. In this case, the average bit error rate (BER) in the high SNR regime is easy to characterize: the BER is proportional to $\mathrm{SNR}^{-\mathrm{D}}$. Figure 8 illustrates the effect of diversity order on BER for a simple binary modulation scheme in AWGN. Note that the error rate can be drastically decreased through the exploitation of higher diversity orders. Doing so, however, often requires complicated receiver processing. Thus, practical systems are usually designed according to a cost/performance tradeoff which captures some, but not all, of the available diversity. 


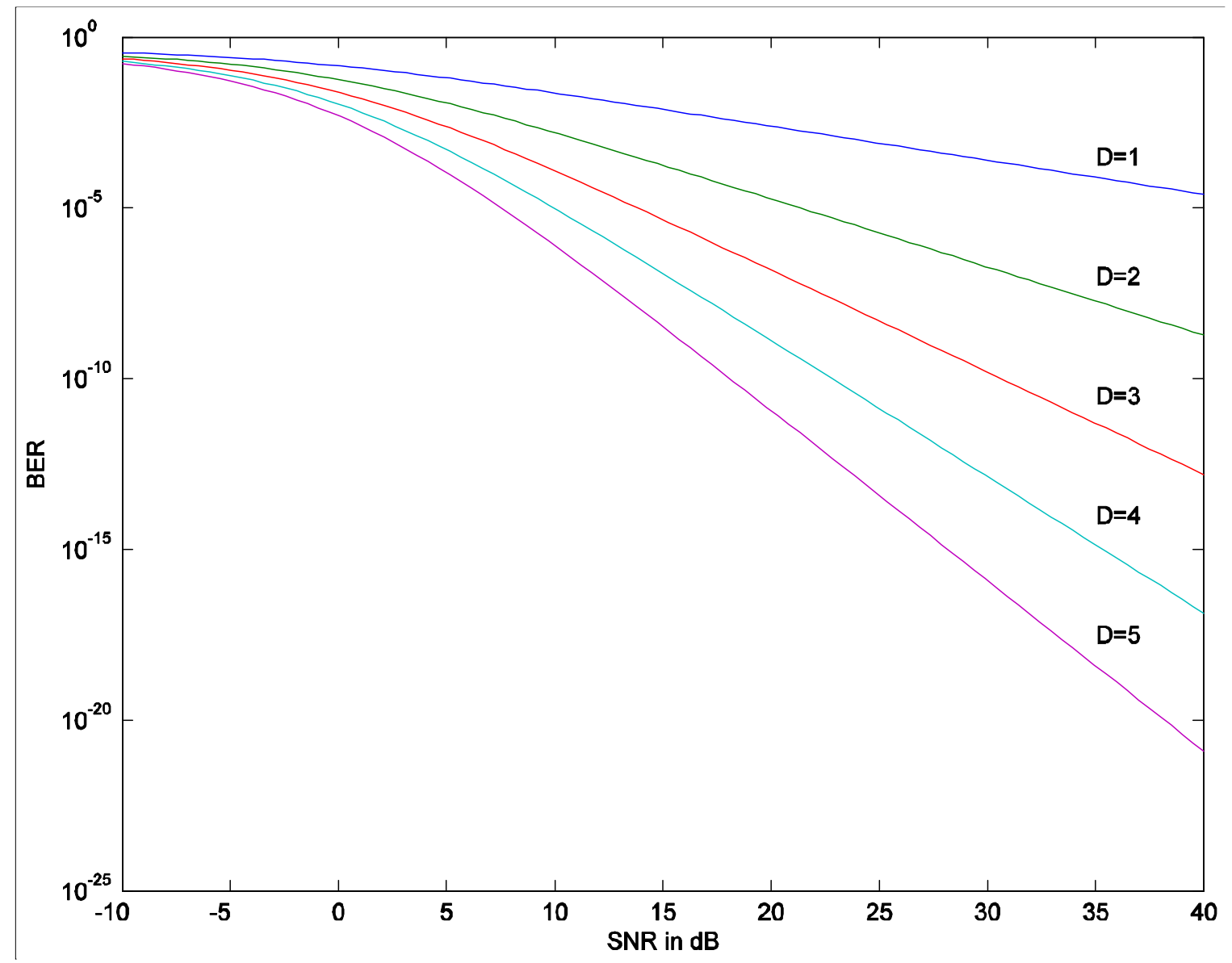

Figure 8: BER of binary phase shift keying over D flat Rayleigh diversity branches in the presence of AWGN.

In a multipath propagation environment, the use of multiple antennas at both transmitter and receiver can be used for spatial diversity gain, as described earlier, or to increase the information rate. To understand the possibility for rate gain, recall that multiple antennas allow beamforming, via which information can be transmitted independently over decoupled propagation paths. In the case that $\mathrm{M}$ antennas are used at both the transmitter and the receiver, it is possible to transmit $\mathrm{M}$ independent bit-streams, each with a spatial diversity order of one. Alternately, we could transmit M/2 independent bit-streams, each with spatial diversity order of two (assuming $\mathrm{M}$ is even). This principle is known as the diversity-multiplexing tradeoff [1].

\subsubsection{Noise}

The term "noise" is typically used to refer to unstructured disturbances which appear like additive random noise at the receiver. There can many sources of noise which, on their own, may be negligible but, together, become significant. Very often, the total noise contribution is well modeled as additive white Gaussian noise (AWGN). Mathematically, the Gaussian approximation can be justified by the central limit theorem [2], which says that the sum of a large number of independent and identically-distributed (but possible non-Gaussian) random 
variables behaves like a Gaussian random variable. Practically speaking, the AWGN approximation is attractive because it simplifies analysis/simulation without compromising much in the way of accuracy. For example, hyper-accurate modeling of individual interferers is seldom worth the effort because, in the end, the sum interference is nearly indistinguishable from AWGN.

Thermal noise generated by the receiver circuitry is said to have a flat power spectral density up to about $1000 \mathrm{GHz}$ [3] and is well modeled as AWGN. Sky noise, including atmospheric and galactic effects, is weakest in the $1-10 \mathrm{GHz}$ range, motivating satellite communication in this band. When the effects of rain are factored in, the sky noise "sweet spot" shrinks to the 2-3 $\mathrm{GHz}$ band. Over any signal bandwidth of several $\mathrm{MHz}$, however, the sky noise density will appear relatively flat and is well modeled by AWGN. Residual ${ }^{4}$ self-interference (e.g., residual ISI) and residual multiple access interference (e.g., from out-of-cell co-channel users) are usually modeled as AWGN as well. The effects of mild receiver distortions (e.g., local oscillator phase noise, power amplifier nonlinearities, and inter-modulation distortion) can also be modeled as AWGN; though technically signal-dependent, these distortion components become, in many cases, uncorrelated from the signal as a consequence of the non-linear distortion mechanism.

Situations where the additive noise is not well modeled by a Gaussian distribution are possible, though generally infrequent, in wireless communication. In contrast, power-line (wired) communication is known to suffer significantly from impulse noise generated by, e.g., electric machinery [4]. It is conceivable, however, that power lines or nearby electric machinery could radiate enough impulse noise to be picked up by a wireless system. For example, an automobile's alternator can sometimes affect the operation of its radio.

\subsubsection{Interference}

The term "interference" typically refers to structured disturbances. Well designed communication systems exploit the structure of the interference when mitigating its effects. Examples of interference include self-interference, multi-access interference, co-channel interference, adjacent channel interference, and intentional jamming.

Self-interference comes in several forms. As discussed earlier, the time-spreading effect of multipath propagation (i.e., frequency-selective fading) will induce inter-symbol interference (ISI) when the delay spread is larger than the symbol interval. With relative motion between the transmitter, receiver, and scatterers, different paths can experience different Doppler shifts, in which case multipath propagation leads to frequency spreading. Frequency spreading will induce inter-carrier interference (ICI) in multi-carrier systems with closely spaced subcarriers. In high spectral efficiency wireless systems, it is not unusual for self-interference to be the dominant impairment, requiring sophisticated "channel tracking" and "equalization" at the receiver.

Multi-access interference (MAI) occurs when a system shares a frequency band simultaneously among several users. Examples include wireless voice and data (e.g., 802.11) systems.

\footnotetext{
${ }^{4}$ By "residual interference," we mean that which remains after the receiver does its best to remove the structured interference.
} 
The mitigation of MAI can be accomplished through multi-user detection, [5] where all users' streams are jointly demodulated at each terminal, or through single-user detection, where the statistical structure (e.g., power spectrum) of the MAI is exploited for its suppression.

Co-channel interference (CCI) occurs when different communication systems share the same bandwidth. For example, terrestrial digital television systems are sometimes operated in the same frequency channels as legacy analog systems. Co-channel systems are usually designed so that the CCI can be easily suppressed. For example, if the legacy system invests most of its transmission energy in a sinusoidal carrier, then it would be wise for the modern system to avoid investing a significant portion of its transmission energy at that carrier frequency.

Adjacent channel interference (ACI) results when two communication systems in neighboring frequency bands interfere. While most systems are designed to minimize ACI, filters with steep roll-offs are expensive, forcing a compromise between cost and spectral efficiency. ACI mitigation can leverage the fact that the ACI spectrum will be skewed towards the band edges.

Jamming, unlike the interferences discussed previously, is an intentional form of interference. The goal of jamming is to deny reliable communication to an adversary's system, and to do so with minimum cost. Jamming signals can be concentrated in time, in frequency, in both time and frequency, or in neither (i.e., spread over a large time-frequency range). Given a finite energy, however, a jamming signal becomes less potent as it is spread wider in time and frequency. Generally speaking, a good jammer will place as much energy as possible at the precise times and frequencies used by the adversary's communication system. Conversely, a jam-resistant system will make it hard for the jammer to know where to invest his energy.

\subsection{Strategies for Reliable Communication}

Section 2.2 discussed multipath fading, noise, and interference: the channel impairments most likely to affect wireless communication. In this section, we describe various modulation schemes and discuss how they are affected by these impairments.

Together, coding and modulation determine how the information bit-stream is translated into waveforms for transmission over the wireless channel. There are many factors which influence the choice of coding/modulation for a particular application (recall the discussion in Section 2.1.1). Speaking very broadly, the choice of coding/modulation reflects a tradeoff between efficient utilization of wireless channel resources (e.g., space, time, bandwidth, power) and robustness to wireless channel impairments (e.g., fading, self-interference, external noise/interference). 


\subsubsection{Channel Capacity}

The notion of "efficient utilization of wireless channel resources" follows from physical limitations on the rate of reliable communication. We now discuss some of these limitations for the simple case of a point-to-point link with a single antenna at each end, a signaling duration of $\mathrm{T}$ seconds, and a transmission bandwidth of W Hertz. Given these time-bandwidth constraints, it is possible to construct approximately WT "orthogonal" (i.e., non-interfering) waveforms. On each of these waveforms, it is possible to transmit information that does not interfere with that transmitted on the other waveforms. Clearly, then, the total amount of information that can be transmitted reliably across this channel increases with the time-bandwidth product W T . Going further, we note that each of these orthogonal waveforms could be transmitted at various amplitudes/phases to communicate multiple bits of information. But there is a tradeoff here. Constraints on transmitted power limit the amplitude range, so that squeezing many bits onto each orthogonal dimension requires creating a set of waveforms that differ only marginally in amplitude/phase. This, in turn, reduces robustness against noise/interference.

This rate/reliability tradeoff turns out to be fundamental. In the late 1940's, Claude Shannon showed that it is theoretically possible to transmit up to $W T \log _{2}\left(1+\frac{P}{W N_{0}}\right)$ bits with arbitrarily low probability of error, where $\mathrm{P}$ denotes the received power and $N_{0}$ the noise spectral density [6]. Shannon's formula has several implications, two of which are illustrated in Figure 9. In Figure 9(a) the maximum spectral efficiency, $\log _{2}(1+S N R)$ of the AWGN channel is plotted as a function of $S N R=\frac{P}{W N_{0}}$, while in Figure 9(b) the maximum channel capacity, $W \log _{2}\left(1+\frac{P}{W N_{0}}\right)$, is plotted as a function of bandwidth $W$ under a fixed power $P$ and noise density $N_{0}$. Spectral efficiency is an appropriate metric for applications which are primarily bandwidth limited. There the goal is to maximize information rate (in bits/sec) per Hertz of precious bandwidth. In this case, Figure 9(a) shows that the information rate grows logarithmically with SNR when the SNR is above a threshold of about $2 \mathrm{~dB}$. For large transmission bandwidths, however, maintaining $\mathrm{SNR}>2 \mathrm{~dB}$ may require unreasonable amounts of transmission power. In this case, it may be more appropriate to fix the total power at $P$ and analyze the effect of bandwidth $W$. Figure 9(b) shows that, when the total power is fixed, there exists a bandwidth above which the system becomes primarily power limited (i.e., allocating additional bandwidth does not appreciably increase capacity). 

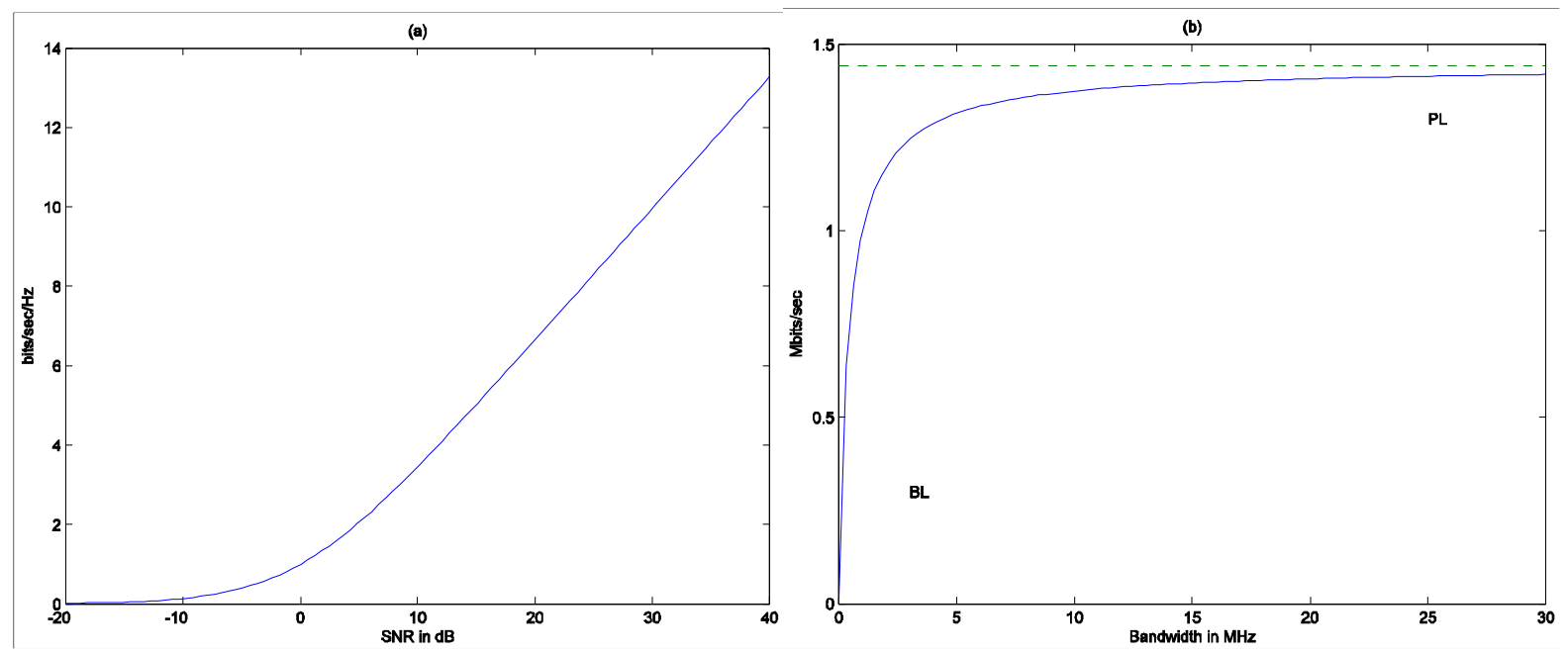

Figure 9: (a) Maximum spectral efficiency (bits/s/Hz) of the AWGN channel versus $S N R=\frac{P}{W N_{0}}$. (b) Capacity (Mbits/s) versus bandwidth $\boldsymbol{W}(\mathbf{M H z})$ when $\frac{P}{N_{0}}=10^{6}$. The dashed line indicates limit as $W \rightarrow \infty$.

Shannon's capacity formula is theoretical in that it shows what is possible with an "infinitely complex" coding/decoding scheme, which requires an infinite amount of time and/or bandwidth for communication and a receiver of infinite complexity. Fortunately, "practical" coding/decoding schemes now exist which come "close" to achieving Shannon's bounds over the AWGN channel. However, most practical channels are dominated by some form of non AWGN interference, such as multi-access interference, self-interference (e.g., ISI or ICI), and colored external interference (e.g., CCI, ACI, or jamming). In the remainder of this section, we discuss the effects of various forms of structured (i.e. non-AWGN) interference on particular modulation schemes.

\subsubsection{Self Interference}

Self-interference manifests when multipath propagation causes several time-frequency shifted copies to impinge on the receiver. When multiple antennas are used, self-interference can have a spatial component as well. The extent of the interference is strongly affected by the modulation scheme in use. Figure 10 gives a graphical depiction of several modulation schemes.
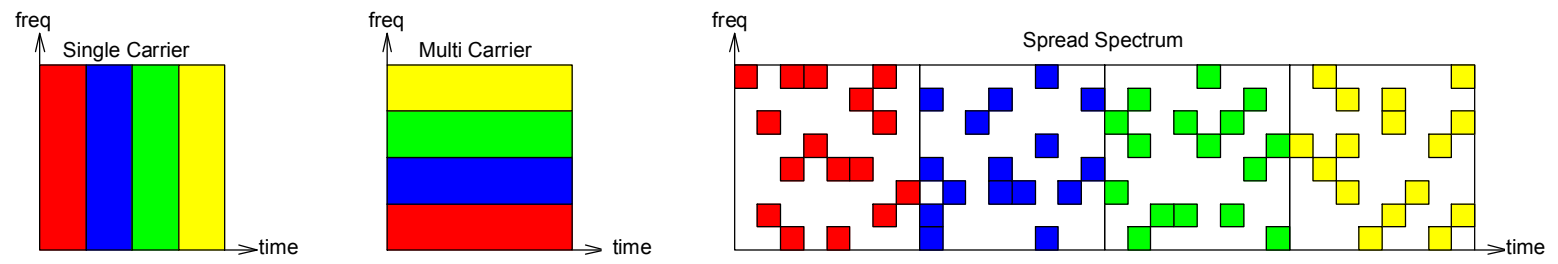

Figure 10: Graphical representation of various modulation schemes. Each colored block represents that time-frequency support of a different symbol. 
In single-carrier schemes, a sequence of symbols is transmitted such that each symbol occupies the full signaling bandwidth (see Figure 10). In high data rate single-carrier systems, the symbol interval may be quite small. When the symbol interval is less than the channel's delay spread, multiple symbols will overlap at the receiver, resulting in inter-symbol interference (ISI). In some systems (e.g., North American digital terrestrial television) the ISI can span several hundred symbols, mandating very complex equalization at the receiver. In the time domain, equalization can be thought of as a method to "de-echo" the received signal. In the frequency domain, equalization can be thought of as a filtering operation that attempts to invert the frequency-selective channel.

Multi-carrier schemes have been proposed to reduce receiver complexity in large-delayspread channels. There, the bandwidth is divided into sub-channels, each which carries only a fraction of the total data rate. By reducing the sub-channel data rate, it is possible to increase the subchannel symbol interval beyond the channel's delay spread, thereby eliminating ISI (see Figure 10). The danger, however, is that channel time-variation can induce frequency-domain spreading which may lead to inter-carrier interference (ICI), especially if the sub-channels are closely spaced. The design of multi-carrier systems (or the choice to use a single-carrier system) is thus strongly influenced by the extent of the time- and frequency-domain spreading.

Single- and multi-carrier schemes are typically employed in bandwidth-limited applications, where it is very important to squeeze as many bits as possible into a given time-frequency resource. When the allocated bandwidth is much larger than that needed to support the desired data rate, there are advantages to spreading the information over a large time-frequency window. This is accomplished by spread-spectrum schemes. (See Figure 10, where the spread-spectrum system transmits each symbol using a time duration which is four times that of the equivalent single- or multi-carrier system.) For example, by making the signal spectrum mimic low-level AWGN, a spread-spectrum system can be operated in the same time-frequency region as other systems without significantly impacting their performance. With multipath propagation, several time/frequency-shifted copies of the spread spectrum signal will impinge on the receiver. Due to the rich signal structure, these signal copies will be approximately orthogonal, and thus it is easy to pick off one copy and suppress interference from the others. One can do much better, however, by picking off several of the strongest copies and combining them coherently. This process is usually referred to as rake reception.

So far we have focused on single-antenna systems. When multiple transmit antennas are employed, multiple signals will impinge on the receiver - each at a different phase - even in the absence of multipath propagation. Usually, the signals transmitted from each antenna are designed so that they combine conveniently at the receiver; the more the transmitter knows about the channel, the more control it has. When multiple receive antennas are employed, their outputs - which usually differ in phase - must be combined. This brings the possibility of "spatial filtering," i.e., isolation of signal components arriving from particular directions. Once isolated, these components could be nulled or combined (as in rake reception). 


\subsubsection{Multi-access Interference}

In multi-access applications, the finite channel resources must be shared among several users. The most popular ${ }^{5}$ multi-access communication schemes include time-division multiple access (TDMA), frequency-division multiple access (FDMA), and code-division multiple access (CDMA). In TDMA, each user is allocated a time slot in which he can occupy the full bandwidth. In FDMA, each user is allocated a frequency channel which he occupies over all time. In CDMA, each user can occupy all times and frequencies, but in a way that does not overlap with other users. Figure 11 gives a graphical depiction of these multiple access schemes.
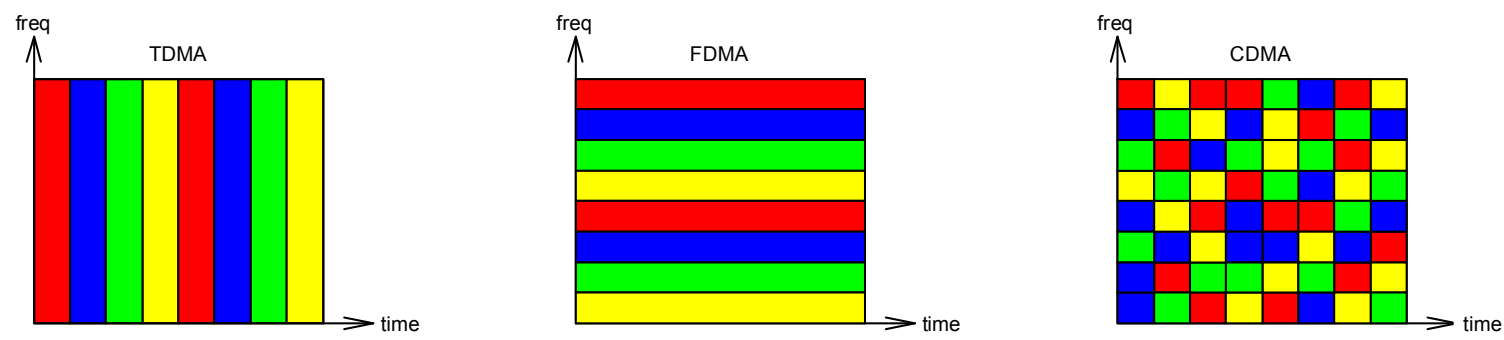

Figure 11: Graphical representations of various multi-access schemes. Each colored block represents the time-frequency support of a different user.

When the dominant channel impairment is AWGN, all three multi-access schemes yield the same performance. In fading or structured interference, however, each scheme has particular advantages and disadvantages. Frequency-selective fades induced by multipath propagation can be very hard on particular FDMA users. Similarly, time-selective fades can be very hard on particular TDMA users. In some cases, coding can be used to "average" the effects of fading, while in other cases the system's delay (or bandwidth) constraints prevent coding over long enough blocks. ${ }^{6}$ In contrast, the time-frequency spread of CDMA signals distributes the effects of fading evenly among CDMA users, which can be very advantageous.

When in the presence of time-domain dispersion (caused by multipath propagation or imperfect time synchronization), TDMA systems may require time-domain guard intervals to prevent multi-access interference (MAI). Similarly, in the presence of frequency-domain dispersion (caused by a time-varying channel or imperfect inter-user frequency synchronization), FDMA systems may require frequency-domain guard bands to prevent MAI. MAI is practically unavoidable in CDMA systems because their users are interwoven tightly in time and frequency. As a result, these systems rely on complicated MAI-cancellation at the receiver and/or sophisticated power-control at the transmitter to ensure that each stream is just strong enough to be demodulated by the intended recipient, but no stronger, since this would increase the MAI seen by other users.

In conclusion, TDMA, FDMA, and CDMA systems are all sensitive to the effects of fading and non-AWGN interference, though in different ways. TDMA/FDMA systems result in strong

\footnotetext{
${ }^{5}$ For example, terrestrial television and radio are based on FDMA, the IS-136 and GSM cellular standards are based on TDMA, while the IS-95 cellular standard is based on CDMA.

${ }^{6}$ The GSM system design tradeoffs provide an interesting example of this concept [1, p. 69].
} 
point-to-point links, implying simple transmitter/receiver designs but difficult cell-site planning/optimization. CDMA systems result in just-adequate point-to-point links, requiring more complex transmitter/receiver designs but simpler cell-site planning/optimization. See [1, Ch. 4] for a more thorough discussion.

\subsubsection{External Interference and Jamming}

As discussed in Section 2.2.3, external interference comes in several forms. To ascertain how significantly an interferer will affect a modulation scheme, one looks to see how closely the interference and transmitted spectra match. For example, if the modulator transmits an information packet in a frequency band where the interference is strong, we expect significant degradation of that packet. By the same principle, a time-concentrated burst of noise could severely degrade an information packet that was transmitted in a time-concentrated manner. It is usually wise to spread each information packet over a large time-frequency range because the robustness to time- or frequency-concentrated interference is greatly enhanced. In other words, "don't put all your eggs in one basket."

For these reasons, CDMA is typically considered as the multi-access system most robust to external interference. Among single-user transmission schemes, spread-spectrum is the most robust. Recall that spread-spectrum robustness comes at the price of reduced spectral efficiency, however (see Figure 10). Here again we see a manifestation of the "rate/reliability tradeoff."

The aforementioned principles apply to jamming as well as unintentional interference. For maximum effect, a jammer will place as much energy as possible at the precise times and frequencies used by the adversary's communication system. If the adversary is using a single- or multi-carrier scheme, it is relatively easy to measure the occupied times or frequencies and then jam accordingly. If the opponent is using a spread spectrum system, however, it is much more difficult to learn the precise time/frequency pattern occupied by the system. When the jammer does not know the spreading pattern, it has several options, none of which are very effective. For example, spreading the jamming signal out in time-frequency will be ineffective because most of the jamming energy will be wasted on inactive signaling dimensions. Concentrating the jamming signal at a particular time or frequency will also be ineffective since the spreadspectrum system is robust to this type of interference. If the jammer knows the signaling pattern, however, it would be easy for him to invest all the jamming energy into the precise timefrequency locations used for signaling. For this reason, jam-resistant spread spectrum systems carefully guard their signaling patterns. Frequency-hopped spread spectrum (FHSS) systems attempt to stay one step ahead of the jammer by quickly changing the transmission frequency according to carefully-guarded pseudo-random sequence. Discrete-sequence spread spectrum (DSSS) systems transmit at many frequencies simultaneously, but according to a carefullyguarded and quickly-varying pseudo-random pattern. Multiple receiver antennas can also be used to counter the effects of jamming: nulls can be placed in the direction of jamming signals.

\subsection{What Matters in a Simulation}

In Sections 2.2 and 2.3, we examined the essential characteristics of the wireless propagation environment and discussed the effects of these characteristics on specific wireless 
communication schemes. This section presents an itemized summary of these essential characteristics from the perspective of simulating a wireless communication system.

The (near) exact modeling of electromagnetic wave propagation in complex physical environments would be incredibly complex. Fortunately, exact modeling is not necessary, since a subset of propagation characteristics suffices to predict communication system performance. In modeling the wireless communication channel, we aim to include all important channel features while purposely omitting those non-important characteristics that unnecessarily consume resources (e.g., compute time, memory, labor-intensive specification, etc.).

As discussed in Section 2.2, the essential propagation characteristics - i.e., multipath propagation, noise, and structured interference - can vary dramatically over time, frequency, and space. In other words, the multipath/noise/interference characteristics at a particular time/frequency/location will rarely, if ever, match those at different times/frequencies/locations, and hence must be taken with a grain of salt. Because the exact channel parameter trajectories are impossible to predict, it is customary to model them as random processes and characterize the statistically average communication system performance. The essential propagation characteristics are then described by the parameters of the statistical models, e.g., the means, covariances, and cross-covariances of Gaussian random processes. In many cases, this statistical approach can be justified rigorously by the Central Limit Theorem and the Law of Large Numbers [2].

\subsubsection{Multipath Fading Parameters}

Here we summarize important statistical parameters which pertain to multipath fading. A more extensive discussion of these concepts can be found in several books $[7,8,9,10]$. Recall that multipath fading can lead to significant levels of self-interference (e.g., ISI, ICI) which mandates proper treatment at the receiver for successful data demodulation.

- Mean received signal power describes the average signal energy reaching the receiver, where averaging is performed globally over time and frequency, and locally over location. Location averaging can be adjusted to highlight either the large scale or shortscale relationship between received power and transmitter/receiver separation, as shown in Figure 12.

The "proper" level of detail in the mean power response depends on how much local averaging is inherently accomplished in data communication. For example, if, at a particular location, the received signal strength varies from weak to strong over a data packet's duration, then (with appropriate coding) the effective signal strength of that packet will be "moderate." Repeating the same experiment at a slightly different location, we might find that the signal strength over the data packet varies from strong to weak, once again resulting in an overall "moderate" signal strength. Hence, with this communication system, the location-specific variations are masked by temporal averaging. 
If, on the other hand, we repeat these experiments with a shorter packet length and/or inadequate coding, temporal averaging may not come into play. In this case, the entire packet would be received weakly at the first-tested location and strongly at the secondtested location. Notice that these two experiments use the same physical environment; what differs is the manner in which the communication system interfaces with the environment.

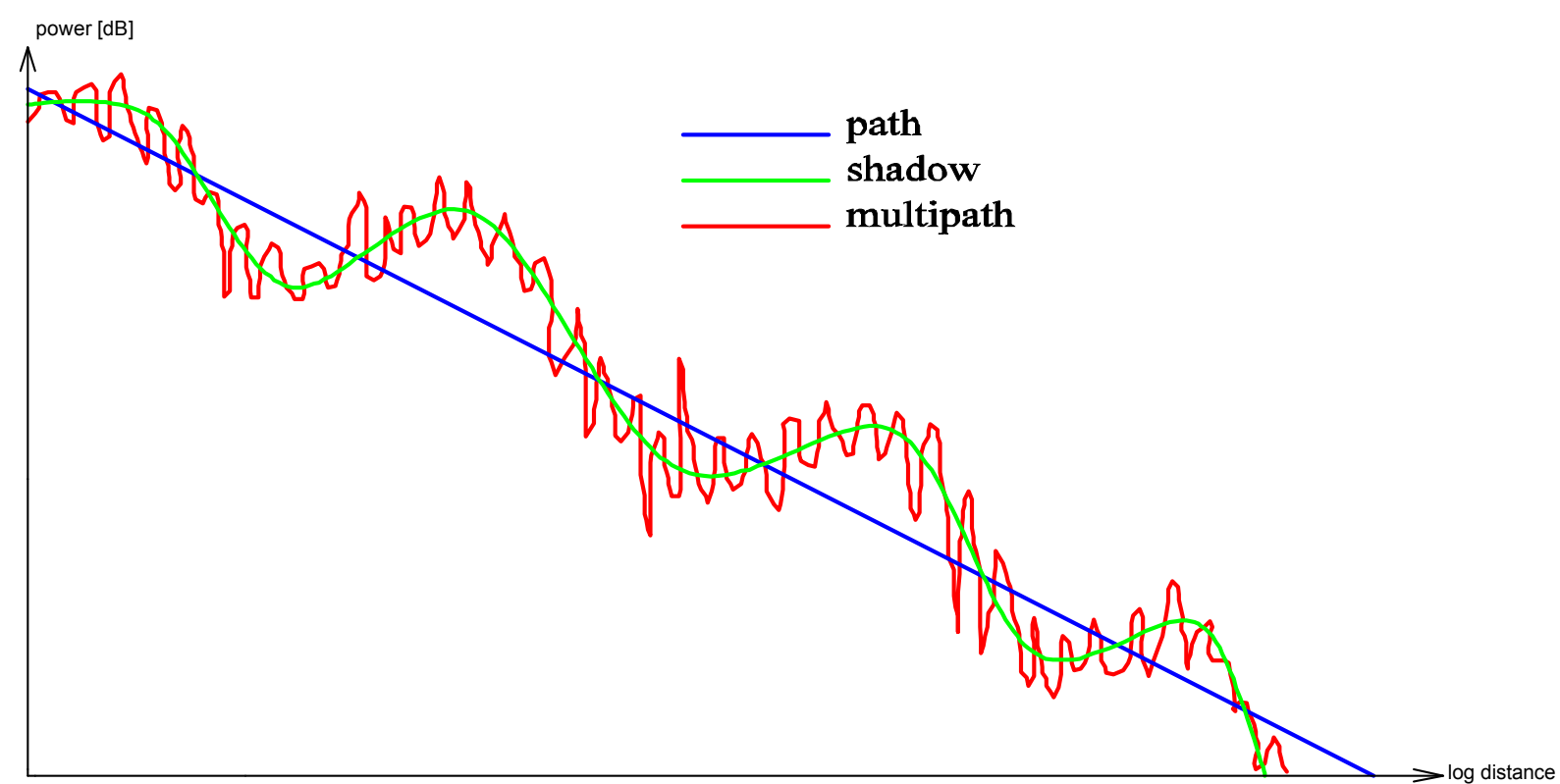

Figure 12: Mean signal power as a function of distance between transmitter and receiver under different amounts of local spatial averaging. (Redrawn from [11].)

The large-scale dependence of signal strength on transmitter/receiver separation is referred to as "path loss" and is modeled by the "path loss exponent" which is said to vary between -2 and -6 .

- The spatial covariance quantifies how quickly the mean received power changes as a function of transmitter/receiver separation. This parameter is important if, e.g., the communication system leverages spatial diversity to combat multipath fading. As discussed in Section 2.2.1, spatial diversity can be exploited through the use of multiple antennas. But it can also be used indirectly, as a form of temporal diversity in even single-antenna systems, when the transmitter and/or receiver locations change with time. The covariance can also be characterized as a function of angle, which can be important if multiple and/directional antennas are used.

- The delay power profile is the statistical description of the multipath delay spread. Specifically, it describes the distribution of energy versus delay. Two of the most commonly assumed delay power profiles are the exponential and uniform profiles shown in Figure 13. The exponential profile is characterized by a decay parameter and the uniform profile by a max delay spread. The exponential profile reflects the tendency for longer paths to experience more path loss, while the uniform profile reflects more of a 
"worst case" setting where the longer paths remain strong (e.g., reflections from distant skyscrapers, water towers, airplanes, or mountain sides).

There are several notions of "delay spread," including, e.g., RMS, mean, 90\%, and maximum delay spreads. Recall that, when the delay spread is much smaller than a symbol interval, i.e., the case of "flat fading," the delay spreading phenomenon becomes insignificant.
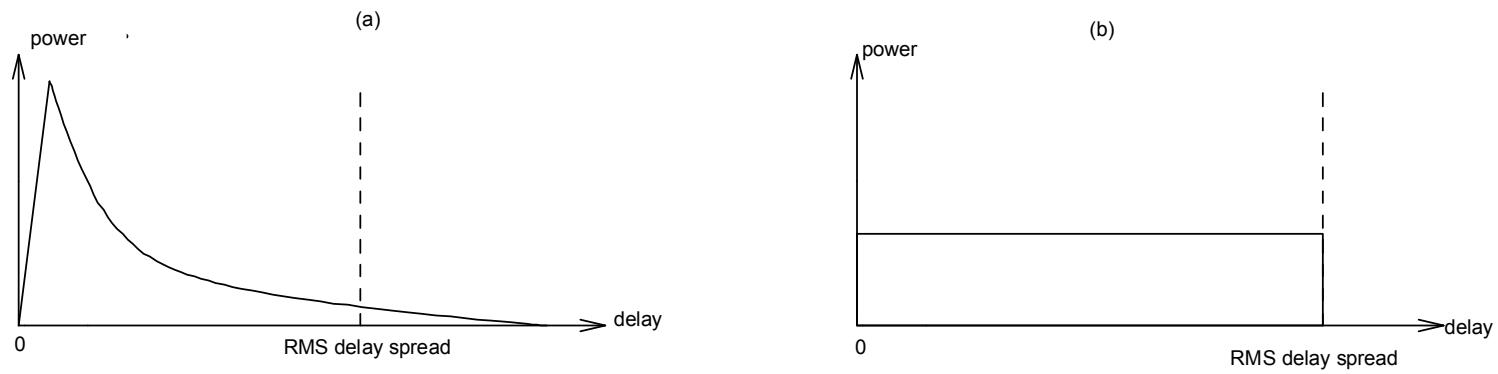

Figure 13: Delay power profiles: (a) exponential, (b) uniform.

- The Doppler power profile is the statistical description of the channel's time variation. In flat fading channels, the Doppler profile is the power spectrum of the complex-valued channel gain. In frequency-selective fading channels, there could be a different Doppler profile for the complex-valued channel gain at each delay. However, it is often assumed that the Doppler profile is independent of delay. Figure 14 illustrates two commonly assumed Doppler power profiles: the "bathtub" profile and the uniform power profile. The bathtub profile describes the situation where the transmitter (or receiver) is in motion and surrounded by a uniform ring of scatterers; it can be efficiently simulated by the socalled Jakes method [7]. Sometimes the bathtub profile is simplified down to a pair of pure tones at the positive and negative maximum Doppler spread frequencies.

The "Doppler spread" typically refers to the maximum (single-sided) frequency deviation due to channel time variation, i.e., the largest frequency component in any of the (timevarying) impulse response coefficients. The maximum Doppler spread is described by the simple formula $v_{\max } f_{c} c^{-1}$, where $v_{\max }$ is the maximum change in path length per second, $f_{c}$ is the carrier frequency, and $\mathrm{c}$ is the speed of propagation.

(a)

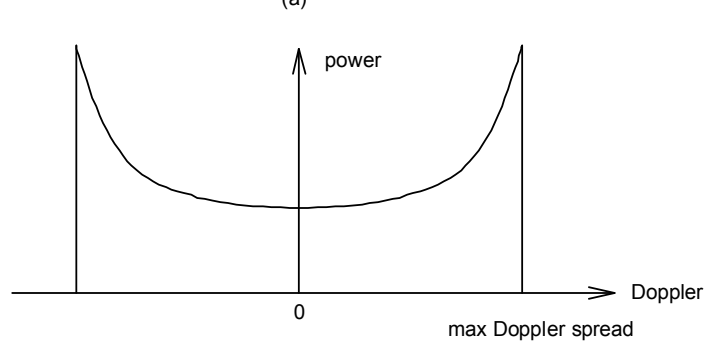

(b)

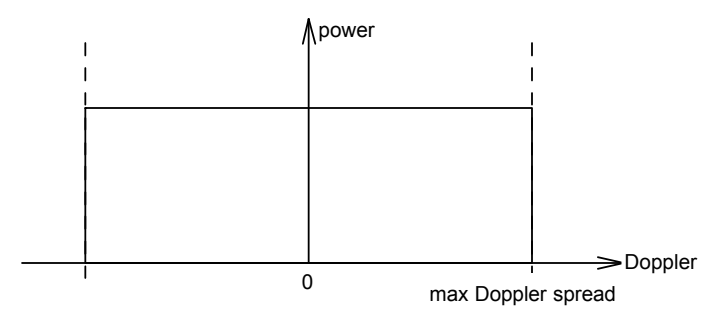

Figure 14: Doppler power profiles: (a) bathtub, (b) uniform. 


\subsubsection{External Noise / Interference Parameters}

External interference comes in unstructured forms (e.g., "noise") and structured forms (e.g., jamming). The relevant statistical descriptions of external interference are discussed here.

- The mean noise-plus-interference power is the overall power of both structured and unstructured interference. For simple expression as a single parameter, it is typically averaged across time, frequency, and location, although - if desired - it could be left as a function of any of these quantities. The signal to interference-plus-noise ratio (SINR), defined as the ratio of mean received signal power to mean noise-plus-interference power, gives a rough indication of the relative signal strength.

Note that reliable communication can be made possible in very-low-SINR environments by sacrificing data rate; spread-spectrum systems are well known for this. Intuitively, the trick is to pack the signal into only a few dimensions of the signaling space, so that the receiver can easily throw away non-signal contributions, thereby increasing the effective SINR.

- The interference power spectrum shows the distribution of interference power across frequency. For example, a narrowband jammer would contribute a peaked component to the interference power spectrum, whereas unstructured (i.e., white) noise contributes a flat component. As discussed in Section 2.3.4, some communication systems are sensitive to peaked interference spectrums.

- When multiple or directional receive antennas are employed, the receiver may be able to exploit the interference direction in interference mitigation. For example, the receiver could steer nulls in those directions which have the lowest SINR.

- While most noise is modeled as Gaussian, some noise has a markedly different statistical distribution which should be modeled as such. Impulsive noise from electric machinery is a common example of strongly non-Gaussian interference. Such signals can be well modeled by a Gaussian mixture, i.e., a noise process that - with high probability - looks like low-variance AWGN, and - with low probability - looks like high-variance AWGN. An interfering digital communication signal can also contribute non-Gaussian interference that can be perfectly removed if the signal can be demodulated. 


\section{Electromagnetic Aspects}

This section addresses the question: How do we obtain the channel parameters of a wireless communications system from electromagnetic (EM) modeling? To answer this question, we must first understand the mechanisms by which wireless communications operate. Then we will consider how to model the various components and how to extract the channel parameters.

\subsection{The Basic EM Components of Wireless Transmission}

Figure 15 shows the basic EM components of a wireless communications system. It consists of a transmitting antenna, a receiving antenna, sources of EM interference, and an EM propagation environment. The transmitting antenna converts a radio frequency (RF) signal from the transmitter into EM waves that travel to the receiver via the propagation medium. The medium may be quite complex and give rise to multipath signals, and may distort the original signal waveform. The receiving antenna converts the EM waves back into an RF signal that goes to the receiver. Interference sources may also generate EM waves at the receiver that corrupt the desired signal. These interference sources may be man made or natural (sky noise). Man made interference may be intentional (jammer) or unintentional.

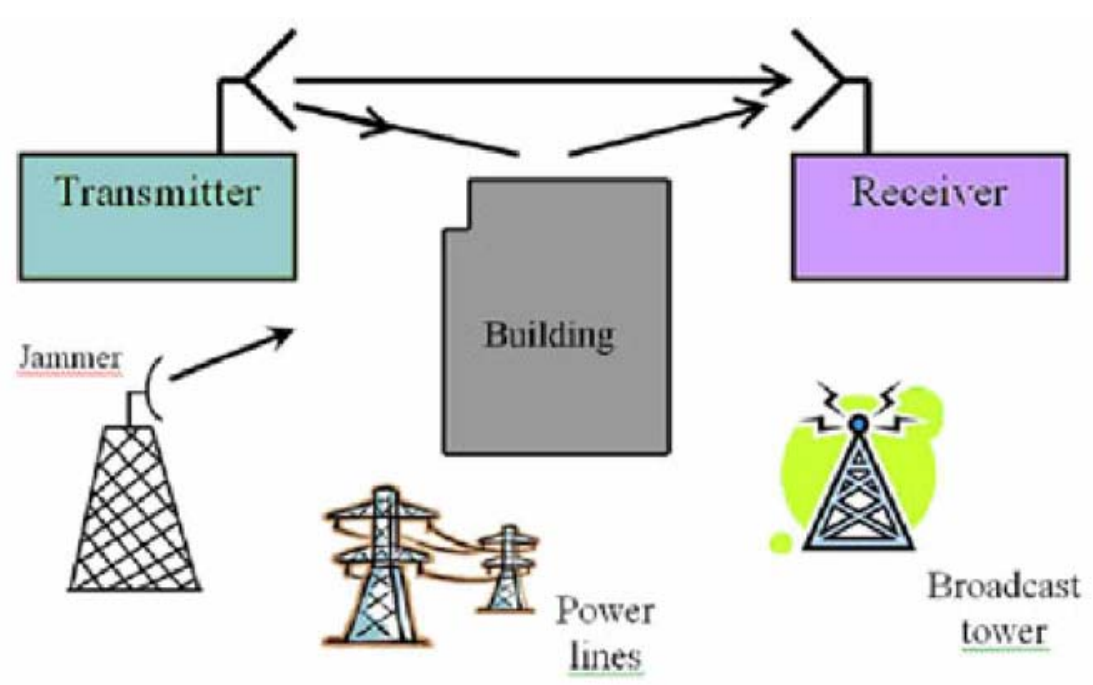

Figure 15: Basic components of a wireless communications system pertinent to EM analysis.

An antenna may act as both transmitter (Tx) and receiver (Rx) in two-way communications, and many systems involve multiple $\mathrm{Tx} / \mathrm{Rx}$ units. Antenna arrays are built up of multiple antennas to achieve spatial selectivity of propagation paths via beamforming and adaptive nulling. Unintentional man made sources of interference may come from electrical lines, machinery, or electronics equipment, where the electrical circuits themselves act as radiating antennas, or from conflicting wireless communications systems with frequency components in the band of interest. Intentional jamming signals are another man made source of interference where the jamming signal is designed to add as much noise and/or signal waveform corruption as possible.

From an EM modeling point of view, there are only two components to consider: the antenna and the propagation environment. These two components may be closely coupled because the 
environment affects the way an antenna radiates, at least in the vicinity of where the antenna is located. Likewise, if there are multiple antennas mounted close together, as in an array, the mutual coupling is important and depends strongly on the platform on which the array is mounted.

It is noted that another EM modeling component may be the RF circuitry feeding the antenna. In fact, printed microstrip antennas are often directly integrated with the RF circuit. In this case the RF circuitry is considered as part of the antenna and may be included in the modeling.

\subsection{Antenna Modeling}

The antenna is the component of a wireless system that can be modeled deterministically, whereas propagation is often modeled statistically. Antennas for wireless systems come in many shapes and sizes. Satellite communications and line-of-sight (LOS) microwave links typically use highly directive reflector or phased array antennas. Cellular base stations use smart antenna arrays to provide spatial selectivity among mobile users. Local wireless networks, mobile cell phones, pagers, two-way radios, etc., use omnidirectional wire or printed circuit antennas. Therefore, different modeling techniques must be considered for each class of antenna.

\subsubsection{Conventional Analysis Methods for Antennas}

Antenna modeling technology is fairly mature. Analysis software is readily available from commercial vendors and DoD software libraries. Engineering formulas are also available in standard antenna textbooks and handbooks such as [12], [13], and [14]. Many antennas, such as wires, microstrip patches, and simple reflectors, are basic enough to characterize from formulas. Furthermore, antennas for wireless communications are often obtained from vendors, and come with a set of specs that sufficiently characterize the antenna.

Antenna modeling codes for small antennas are typically based on numerical methods such as the finite difference time domain (FDTD) method, the finite element method (FEM) or the method of moments (MoM) [15]. Numerical methods are appropriate for wire and printed circuit antennas, as well as antennas integrated into RF circuit boards. These methods have limits based on the electrical size and complexity of the geometry, but are generally very accurate.

Software is also available for modeling large reflector antennas. Reflector codes use highfrequency asymptotic methods such as physical optics (PO), the physical theory of diffraction (PTD), and the geometrical and uniform theories of diffraction (GTD and UTD). Asymptotic methods are more approximate than numerical methods, but can handle much larger geometries. Ray-tracing methods are included in this class of analysis. Asymptotic methods are often combined with numerical methods to model complex feed antennas of reflectors.

Array antennas are more difficult to model because they may be too electrically large for standard numerical methods, and too complex for asymptotic methods. Yet arrays must be carefully analyzed because mutual coupling between antenna elements may significantly affect the input impedance and gain pattern. Sophisticated codes such as ADFMM are being developed 
for this purpose [16]. If the antenna elements are not closely packed together, or are designed to minimize mutual coupling effects, it may be possible to analyze the array elements individually and coherently combine the transmitted or received signal from each element. This latter approximation is often used for base station antenna array modeling and space-time adaptive signal processing.

\subsubsection{Antenna Platform Modeling}

In addition to modeling the antenna, and possibly its RF circuitry, one must consider the platform on which the antenna is to operate. The platform may have a very significant effect on the gain pattern, input impedance and efficiency of the antenna. For example, much work has been done to analyze the effect of the human hand and head on the operation of a cell phone antenna. Likewise, antennas are mounted on aircraft, satellites, ships, and ground vehicles. Therefore, in many cases it is not sufficient to simply analyze the antenna in free space or use vendor specs. Commercial software packages for antennas may not be able to include the effect of a complex platform. Detailed numerical analysis may be necessary in such cases.

If the platform does not significantly affect the radiating currents of the antenna, it is possible to replace the antenna with a set of equivalent currents that radiate as point sources in the presence of the platform. The reciprocity theorem may be invoked to apply the same principle to the receiving antenna [17].

\subsubsection{Interference Source Modeling}

Man made sources of interference can also be modeled as radiating antennas. Intentional sources of interference, and unintentional interference from conflicting wireless systems, are modeled the same way as the system antennas. Other unintentional sources, such as power lines, electronics equipment, and commercial broadcast towers, may not be as straightforward. The modeling of these types of sources can be much more complex than system antennas because one must first understand the radiation mechanisms responsible for the interference. For example, with power lines it may be due to voltage impulses caused by electrical machinery. Electronics equipment may generate interference in a variety of ways, such as from power amplifiers, transformers, and binary switching. Broadcast towers can radiate strong harmonics in the frequency band of interest, even though the carrier frequency is far outside the band.

Because of the many possible causes of unintentional interference, it may be best to approach this problem empirically. If the radiated power spectrum of an interfering source is known, an equivalent source may be substituted in the vicinity of the original source that radiates the same power spectrum. The power spectrum can be obtained from measurements and/or from an analysis of the interference source.

\subsection{Propagation Modeling}

There are two main types of propagation models: empirical and site-specific [18]. Both seek to describe a propagation environment in terms of a small number of statistical parameters. 
Empirical models are for general classes of propagation environments, such as rural, urban, inside an office building, inside a factory, underground, etc. The parameters for empirical models are obtained from past experience, vast databases of measurements, and theoretical analysis. Here we are more concerned with site-specific models where computational EM (CEM) modeling may be used for well-defined geometries. It is, of course, possible to model considerable variation in the geometry by including a large number of cases.

CEM methods come in two basic categories: numerical and analytical. Numerical methods represent an unknown quantity, such as the EM fields or surface currents, in terms of a set of basis functions with unknown coefficients. The unknowns are found by enforcing Maxwell's equations and a set of boundary conditions to obtain a system of equations that is solved numerically. The finite difference time domain (FDTD) method, the finite element method (FEM) and the method of moments (MoM) are all examples numerical methods. They are capable of modeling very complex structures with high accuracy, but can be computationally expensive.

Analytical methods, on the other hand, use closed form solutions of Maxwell's equations. Since realistic propagation scenarios are rarely simple enough to model in closed form, approximate analytical methods have been developed based on ray-tracing. The shooting and bouncing ray (SBR) method, and the geometrical and uniform theory of diffraction (GTD and and UTD) methods all use ray-tracing. Ray methods are much more computationally efficient than numerical methods, but generally not as accurate.

\subsubsection{Numerical Methods}

Purely numerical CEM methods are based on a discretization of the problem geometry and, in the case of finite methods, discretization of Maxwell's equations. Here we will briefly describe the three most popular numerical CEM methods: FDTD, FEM, and MoM. Many commercial software packages have been developed using these methods. A good overview reference for all three methods is the book by Peterson, Ray and Mittra [15].

\section{The finite difference time domain method}

The FDTD method discretizes the differential form of Maxwell's equations in both time and space [19]. Assuming a linear, isotropic, time-invariant, nondispersive media, Maxwell's equations in the time domain are given by,

$$
\begin{aligned}
& \varepsilon(\bar{r}) \frac{\partial \bar{E}(\bar{r}, t)}{\partial t}=\nabla \times \bar{H}(\bar{r}, t)-\bar{J}(\bar{r}, t) \\
& \mu(\bar{r}) \frac{\partial \bar{H}(\bar{r}, t)}{\partial t}=-\nabla \times \bar{E}(\bar{r}, t)
\end{aligned}
$$

where $\bar{E}$ and $\bar{H}$ are the electric and magnetic fields, respectively. $\varepsilon$ and $\mu$ are the dielectric permittivity and magnetic permeability of the media, respectively, and may vary as a function of 
position $\bar{r} . \bar{J}$ is the electric current that may be impressed or induced. An induced volumetric current is related to the electric field by the conductivity $\sigma$ of the medium:

$$
\bar{J}(\bar{r}, t)=\sigma(\bar{r}) \bar{E}(\bar{r}, t)
$$

The FDTD method replaces the derivatives in time and space using the central-difference approximation,

$$
\left.\frac{d f}{d u}\right|_{u=u_{i}}=\frac{f\left(u_{i}+\Delta u / 2\right)-f\left(u_{i}-\Delta u / 2\right)}{\Delta u}+O\left(\Delta u^{2}\right)
$$

where $u_{i}=u_{0}+i \Delta u$. This approximation results in second-order accuracy. Because of this finite-difference approximation, the entire problem domain must be discretized into an orthogonal structured mesh with spacial sample size $\Delta x$. A staggered rectangular grid known as the Yee lattice is often used in 3D FDTD codes [20].

The discretized form of Maxwell's equations are solved by starting with an initial solution at time zero (usually with all quantities set to zero), and stepping through time with a sampling interval $\Delta t$. At each time step the quantities in (3.1) are computed explicitly in terms of the quantities from the previous time steps. This provides a very simple and efficient solution of complex problems.

The stability of the time-stepping solution is governed by the Courant condition. For a 1D problem this condition is,

$$
c \Delta t \leq \Delta x
$$

where $\mathrm{c}$ is the speed of light. This guarantees that information cannot be transmitted faster than the speed of light across the grid. In 3D the Courant condition becomes,

$$
c \Delta t \leq \frac{\Delta x}{\sqrt{3}}
$$

because waves may travel at oblique angles across the mesh.

To set up an FDTD problem, the time step $\Delta t$ is first chosen based on the incident (or impressed) waveform. For example, $\Delta t$ might be chosen as $T / 10$ for an incident pulse of width $T=1 / f_{\max }$, where $f_{\max }$ is the maximum frequency component of the pulse. $\Delta x$ is then chosen to satisfy (3.5), as well as to accurately represent the geometry. It might seem that we should make $\Delta x$ as large as possible to reduce the number of grid points in the mesh. However, this increases the dispersion error. To keep dispersion error under control, $\Delta x$ should also be less than about $\lambda_{\text {min }} / 10$, where $\lambda_{\text {min }}$ is the smallest wavelength in the problem. The smallest wavelength occurs at the highest frequency in the medium with the slowest wave velocity $v_{\min }$, 


$$
\lambda_{\min }=\frac{v_{\min }}{f_{\max }}=\left.\frac{1}{f_{\max } \sqrt{\varepsilon \mu}}\right|_{\min }
$$

Therefore, $\Delta x$ should be chosen in the range,

$$
c \Delta t \leq \Delta x \leq \lambda_{\min } / 10
$$

It is noted that $\Delta t$ may have to be reduced to satisfy this condition. For example, if $\Delta t=T / 10=1 /\left(10 f_{\min }\right)$, then $c \Delta t=\lambda_{0} / 10$ where $\lambda_{0}$ is the free space wavelength. $\lambda_{0}$ is always greater than or equal to $\lambda_{\min }$.

Boundary conditions are imposed on the FDTD mesh wherever it is truncated. As illustrated in Figure 16(a), in an impenetrable enclosure, for example, the boundary condition is specified on the walls and perhaps on waveguide ports that exit the enclosure. Open geometries require an absorbing boundary condition (ABC) to allow waves to pass through as shown in Figure 16(b). The so-called perfectly matched layer is an example of an advanced ABC technique [21]. 


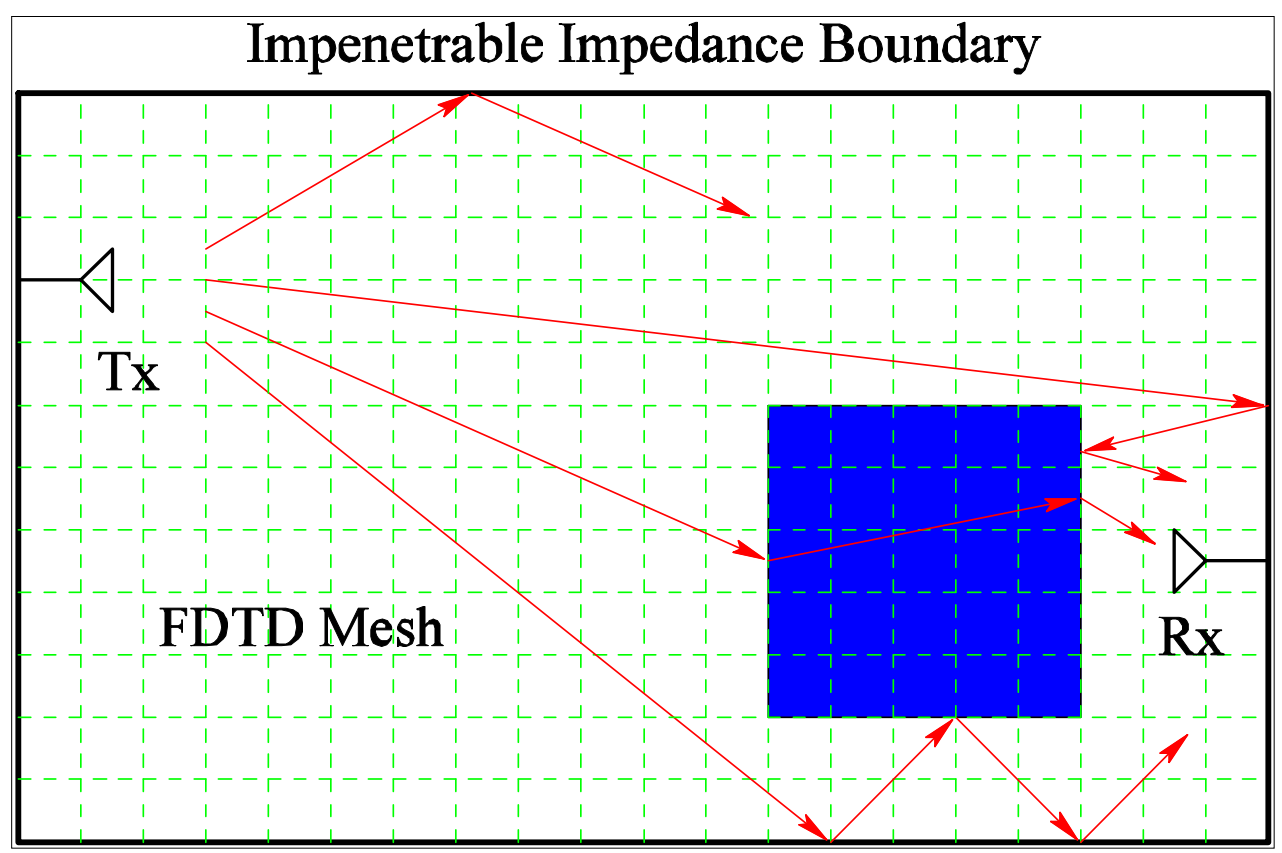

(a) Enclosed geometry.

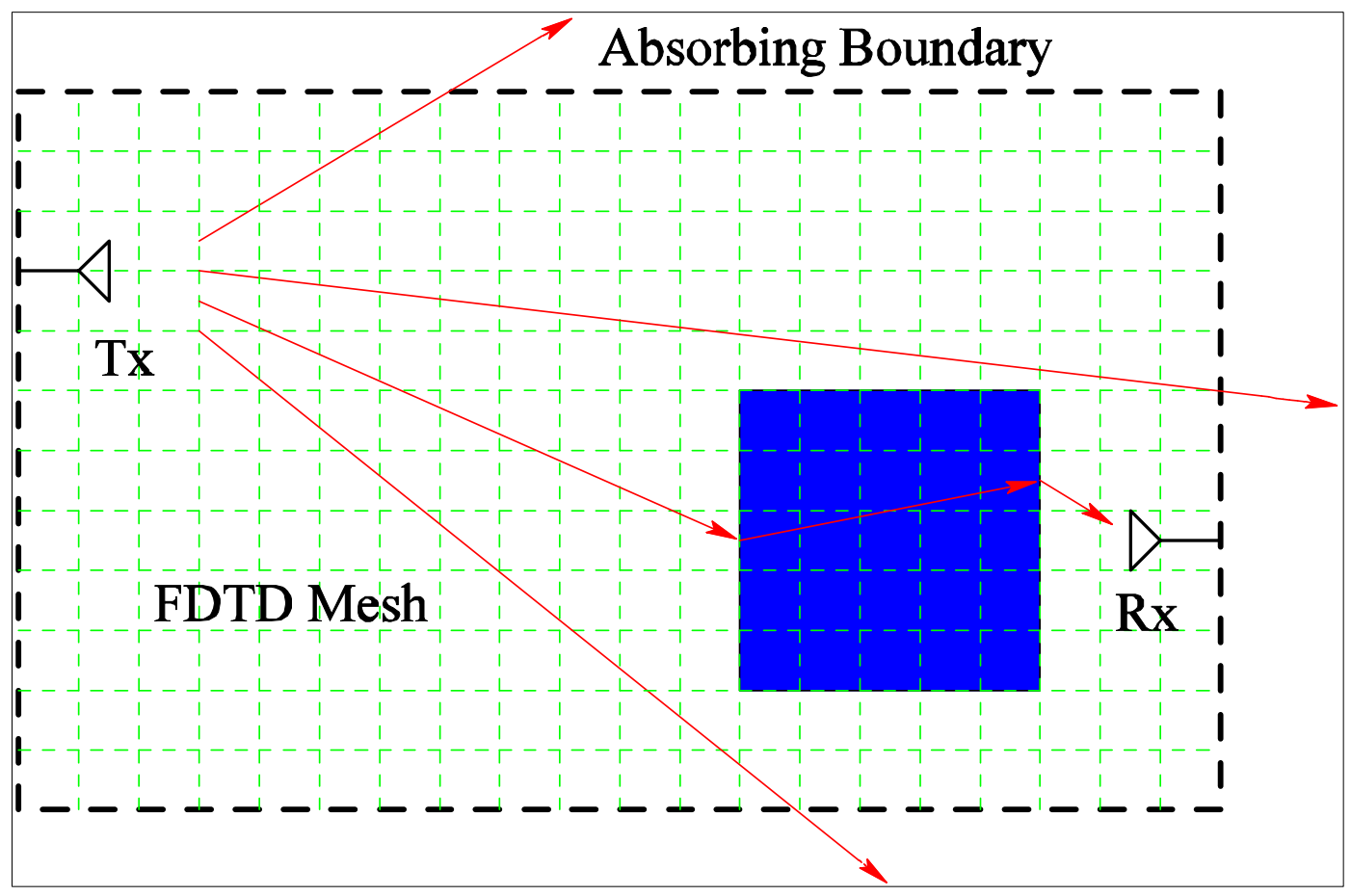

(b) Open geometry.

Figure 16: FDTD problem domains with a structured mesh. 
As seen from Figure 16, the entire volume of the problem domain is meshed, including empty space and any penetrable material regions. The orthogonal mesh, the Courant condition, and dispersion error impose severe limitations on the accuracy and efficiency of the FDTD method. Since the mesh must be uniform, the time step, the smallest geometrical detail, and the material with the slowest wave velocity determine the density of the whole mesh. Furthermore, the entire geometry must be modeled with a stairstep approximation. This leads to accuracy errors if there are a lot of curved or oblique surfaces in the geometry. Therefore, the FDTD method is limited to relatively small problems in terms of wavelength. Recent advances in higher order and adaptive meshing schemes have extended the range of applications somewhat.

In the area of wireless communications, the FDTD method has been very useful for modeling small antennas and objects in the vicinity of the antenna, such as the human hand and head. It is also useful for studying the transmission through specific types of penetrable geometries, such as walls, floors, doorways, and cracks. It is not able to handle wide-area propagation problems unless they are fairly small and localized, such as wireless transmission in a small room or enclosure on the order of 10 wavelengths or smaller. The FDTD is more useful for wideband problems than narrowband because a wideband pulse in the frequency domain is very narrow in the time domain.

Related to the FDTD method is the finite volume time domain (FVTD) method [22]. Without going into detail, the FVTD method utilizes the integral form of Maxwell's equations rather than the finite-difference approximation of the partial differential equations. This has led to extensions and generalizations of the basic FDTD method [15].

\section{The Finite Element Method}

Like the FDTD method, the FEM discretizes the entire volume of the problem domain, but uses an unstructured mesh as shown in Figure 17 instead of an orthogonal mesh [23]. Notice that the mesh may be denser near objects and in materials with higher $\varepsilon$ and $\mu$.

The EM fields are represented using a basis function expansion, usually defined over triangular (2D) or tetrahedral (3D) elements. The solution may be found in the frequency or time domain. Here we will focus on the frequency domain solution. 


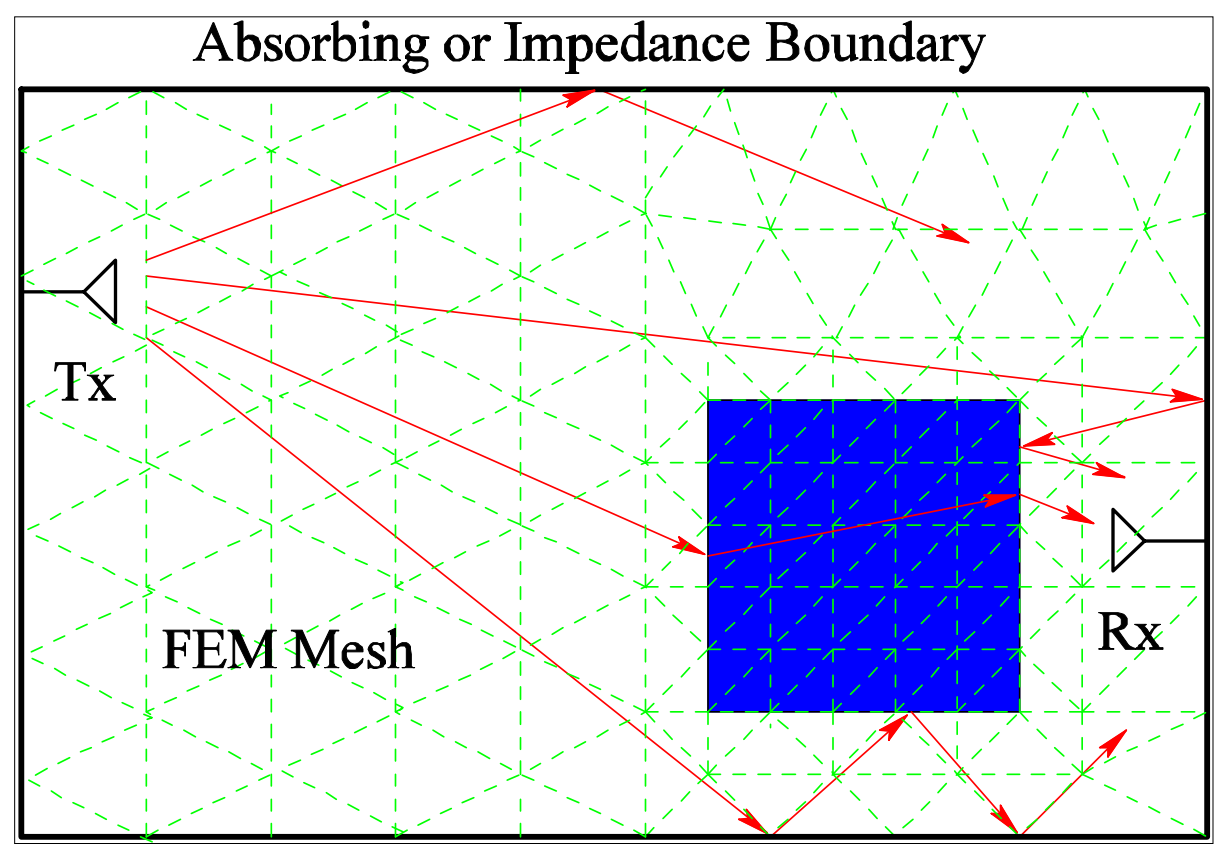

Figure 17: Unstructured FEM mesh in an enclosed volume.

In a source-free isotropic region, Maxwell's equations in the frequency domain may be written as,

$$
\begin{aligned}
& \bar{E}=\frac{1}{j \omega \varepsilon} \nabla \times \bar{H} \\
& \bar{H}=-\frac{1}{j \omega \mu} \nabla \times \bar{E}
\end{aligned}
$$

where $\omega=2 \pi f$ and $\bar{E}, \bar{H}, \varepsilon$ and $\mu$ are functions of position $\bar{r}$ and frequency $f$. Taking the curl of the first equation and substituting it into the second, and taking the curl of the second equation and substituting it into the first results in the vector Helmholtz equations,

$$
\begin{aligned}
& \nabla \times\left(\frac{1}{\mu_{r}} \nabla \times \bar{E}\right)=k^{2} \varepsilon_{r} \bar{E} \\
& \nabla \times\left(\frac{1}{\varepsilon_{r}} \nabla \times \bar{H}\right)=k^{2} \mu_{r} \bar{H}
\end{aligned}
$$

where $k=\omega / c$ is the free space wavenumber, and $\varepsilon_{r}=\varepsilon / \varepsilon_{0}$ and $\mu_{r}=\mu / \mu_{0}$ are the relative material permittivities and permeabilities, respectively. Next we take the dot product of Eqs. (3.9) with a testing function $\bar{T}$ and integrate over the entire volume $\mathrm{V}$ domain of the problem. After manipulation with some vector calculus identities and the divergence theorem, we arrive at 


$$
\begin{aligned}
& \int_{V}\left[\frac{1}{\mu_{r}}(\nabla \times \bar{T}) \cdot(\nabla \times \bar{E})-k^{2} \varepsilon_{r} \bar{T} \cdot \bar{E}\right] d V=-\int_{S}\left[\frac{1}{\mu_{r}} \bar{T} \cdot \hat{n} \times(\nabla \times \bar{E})\right] d S \\
& \int_{V}\left[\frac{1}{\varepsilon_{r}}(\nabla \times \bar{T}) \cdot(\nabla \times \bar{H})-k^{2} \mu_{r} \bar{T} \cdot \bar{H}\right] d V=-\int_{S}\left[\frac{1}{\varepsilon_{r}} \bar{T} \cdot \hat{n} \times(\nabla \times \bar{H})\right] d S
\end{aligned}
$$

where $\mathrm{S}$ is the surface bounding $\mathrm{V}$ and $\hat{n}$ is the outward-pointing unit surface normal of $\mathrm{S}$. Notice that the surface integrals on the right-hand-side may be used to specify the boundary conditions on S. Like the FDTD method, an impenetrable impedance or absorbing boundary condition may be imposed.

Either of Eqs. (3.10) may be solved by FEM by expanding the unknown quantity ( $\bar{E}$ or $\bar{H}$ ) into a set of $\mathrm{N}$ basis functions, along with an impressed excitation function, and then choosing $\mathrm{N}$ testing functions to obtain an N X N system of equations. For efficiency and generality, overlapping subsectional basis functions are usually defined on each pair of interfacing tetrahedral volume elements. The testing functions may be chosen the same way.

Notice that the curl-curl operations in (3.9) have been reduced to single-curl operations by transferring one of the operations onto the testing functions in (3.10). This allows linear basis functions to be easily implemented because the second derivative does not have to be computed. With a choice of linear overlapping subsectional basis and testing functions, it is seen that the volume integrals in (3.10) will be non-zero only for adjacent elements. This results in a sparse or banded system matrix, which allows a very large number of unknowns to be solved efficiently.

Similar to FDTD, FEM requires a mesh density of at least 10 elements per wavelength to control the dispersion error. However, it can handle somewhat larger problems than FDTD because the unstructured mesh is more easily adaptable to the details of the geometry. A major limitation of FEM is that the dispersion error increases with the size of the geometry. This is because the farther waves propagate through a mesh, the more they become distorted. Therefore, the mesh may need to be even denser for larger problems. The FEM has not been exploited as much as FDTD for wireless propagation problems, hence there may be some opportunities for research.

\section{The Method of Moments}

The FEM is technically a subset of the MoM in that basis and testing functions are used to represent the unknown quantity and to convert any operator equation into a system matrix [15]. However, in the electrical engineering community, the name MoM has become associated with the solution of integral equations [24]. Integral equations are based on the radiation of equivalent sources according to a specified Green's function for the background medium. The equivalent sources are unknown quantities that are found by enforcing the boundary conditions of the problem.

Figure 18 illustrates a simple MoM problem. The original geometry is replaced with equivalent

electric surface currents $\bar{J}_{S}$ on the boundary enclosing a homogeneous volume of space. We define the sources to radiate in free space, so the currents and radiated fields therefore 
automatically satisfy Maxwell's equations. This is unlike FEM and FDTD where Maxwell's equations are enforced on an assumed field expansion.

The MoM may also be applied to open radiation problems where the outer boundary shown in Figure 18 extends to infinity. The radiation boundary condition applies at infinity, so we do not need to place equivalent sources there. Therefore, the source Tx and the receiver Rx may be very widely separated.

The fields radiated by the sources $\bar{J}_{S}$ are given by,

$$
\begin{aligned}
& \bar{E}^{s}(\bar{r})=-j k Z_{0} \bar{A}\left(\bar{J}_{s}\right)+\frac{Z_{0}}{j k} \nabla\left[\nabla \cdot \bar{A}\left(\bar{J}_{s}\right)\right] \\
& \bar{H}^{s}(\bar{r})=\nabla \times \bar{A}\left(\bar{J}_{s}\right)
\end{aligned}
$$

where $\bar{A}\left(\bar{J}_{S}\right)$ is the vector potential,

$$
\bar{A}\left(\bar{J}_{s}\right)=\int_{s} \bar{J}_{s}\left(\vec{r}^{\prime}\right) \frac{e^{-j k R^{\prime}}}{4 \pi R^{\prime}} d S^{\prime}
$$

and $R^{\prime}=|\bar{r}-\vec{r}|$. In the above, $\bar{r}$ is the field point and $\vec{r}^{\prime}$ is the source point. The integration is over the primed quantities on the surface S. $Z_{0}=\sqrt{\mu_{0} / \varepsilon_{0}}$ is the impedance of free space.

For a perfectly conducting surface the boundary conditions on $\mathrm{S}$ are,

$$
\begin{gathered}
\left.\bar{E}\right|_{\tan }=\bar{E}^{i}+\left.\bar{E}^{s}\right|_{\tan }=0 \\
\bar{J}_{s}=\hat{n} \times \bar{H}=\hat{n} \times \bar{H}^{i}+\hat{n} \times \bar{H}^{s}
\end{gathered}
$$

where $\bar{E}^{i}$ and $\bar{H}^{i}$ are the incident (impressed) fields. Along with (3.11), Eq. (3.13) is known as the electric field integral equation (EFIE) and (3.14) is the magnetic field integral equation (MFIE). The EFIE may be applied to both open and closed surfaces, but the MFIE is for closed surfaces only. 


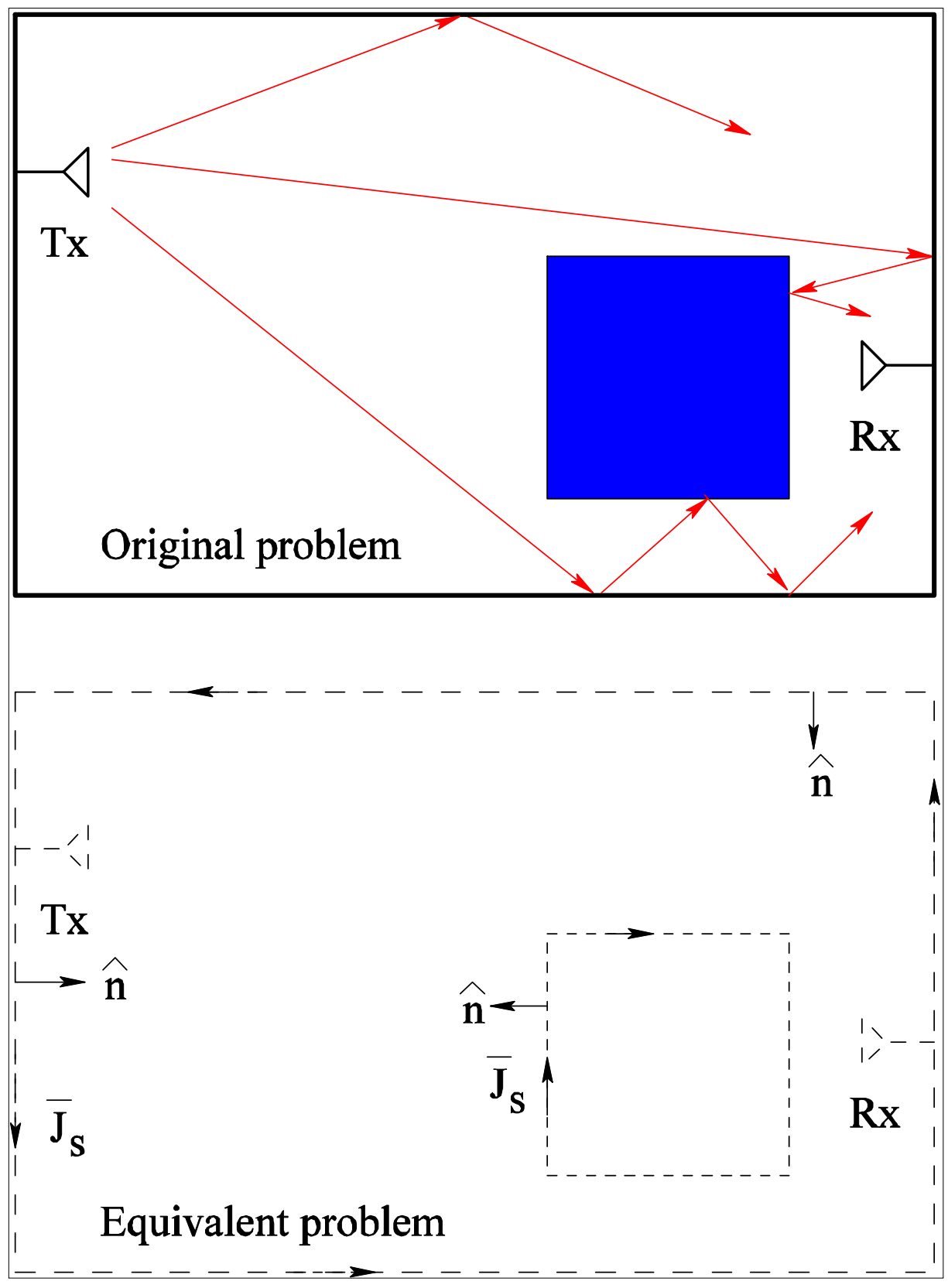

Figure 18: Equivalent source problem for method of moments. 
Next we take the dot product of a testing function $\bar{T}(\bar{r})$ with Eqs. (3.13) and (3.14) and integrate over the surface $\mathrm{S}$. We choose $\bar{T}$ to be everywhere tangential to the surface since $\bar{J}_{s}$ is tangential. After manipulation with some vector calculus identities and the divergence theorem we arrive at,

EFIE:

$$
\begin{aligned}
\int_{s}\left[j k Z_{0} \bar{T} \cdot \bar{A}\left(\bar{J}_{s}\right)\right. & \left.+\frac{Z_{0}}{j k}(\nabla \cdot \bar{T}) \nabla \cdot \bar{A}\left(\bar{J}_{s}\right)\right] d S \\
& -\oint_{L}\left[\nabla \cdot \bar{A}\left(\bar{J}_{s}\right)\right] \bar{T} \cdot(\hat{l} \times \hat{n}) d l=\int_{s} \bar{T} \cdot \bar{E}^{i} d S
\end{aligned}
$$

MFIE:

$$
\int_{s}\left[\bar{T} \cdot \bar{J}_{s}-\bar{T} \bullet \hat{n} \times \nabla \times \bar{A}\left(\bar{J}_{s}\right)\right] d S=\int_{s} \bar{T} \bullet \hat{n} \times \bar{H}^{i} d S
$$

where $L$ is the contour around $S$ if $S$ is an open surface. $L$ is zero for a closed surface.

$\hat{l}$ is the unit tangent vector along $L$. Notice that in (3.15) one of the $\nabla$ operations on $\bar{A}$ from (3.11) has been transferred to $\bar{T}$. This removes an order of singularity from $\bar{A}$ and allows linear basis and testing functions to be easily implemented. It is also convenient to define basis and testing functions that are tangential to edges so that $\bar{T} \cdot(\hat{l} \times \hat{n})=0$ in the contour integral of (3.15). Linear "rooftop" basis functions known as RWG (Rao-Wilton-Glisson) basis functions satisfy this condition and are very popular for expanding and testing currents on 3D triangular meshed surfaces [25].

Looking at Eqs. (3.15) and (3.16) we see that the MoM will give rise to a dense system matrix even when subsectional basis functions are used. This is because the vector potential $\bar{A}\left(\bar{J}_{s}\right)$ is non-zero everywhere even though the basis function for $\bar{J}_{s}$ may be very localized. Therefore, the MoM cannot handle as many unknowns as the FDTD or FEM, but its great advantage is that only the surface needs to be discretized. The sampling density needs to be at least 8 elements per wavelength.

The MoM is able to handle somewhat large problems than FDTD or FEM. Accelerated versions such as the fast multipole method (FMM) have extended the range of applications considerably [26]. Currently it is not unusual for the FMM to handle problems up to 100 wavelengths in size involving more than a million unknowns. This allows fairly large indoor wireless propagation problems to be modeled in the $2.4 \mathrm{GHz}$ band.

\subsubsection{Ray Methods}

Ray methods track the fields radiated by a transmitting antenna along ray paths throughout the propagation environment until they reach the receiver. Rays are the set of lines in space that are normal to equi-phase wavefronts emanating from a simple source. They undergo free space 
propagation, reflection from smooth surfaces, diffuse scattering from rough surfaces, and diffraction from edges to arrive at the receiver. Multiple ray paths from source to receiver are often present in a realistic environment, hence the term multipath. Rays therefore yield much physical insight into the mechanisms by which EM energy propagates.

Figure 19 illustrates the two basic approaches to ray tracking, which are called by different names in the literature. Here we will refer to them as ray-shooting and ray-tracing.

\section{Ray-Shooting}

Here we will define ray-shooting where a large number of rays are launched outward from the source and tracked individually like beams of light until some stopping criteria is reached. This is sometimes referred to as brute force ray-tracing. The shooting and bouncing ray SBR method is an example of ray-shooting [27]. The rays are tracked according to the laws of geometrical optics through multiple bounces until they intersect a reception sphere, as shown in Figure 19(a). Rays that hit a material interface are split into a reflected and refracted ray. Rays that hit an edge are split into a new family of diffracted rays launched from the edge. The fields are calculated at the receiver by the strength and number of rays that hit a pre-defined reception sphere [28].

The reception sphere is not a very robust approach for computing the fields from a set of rays. The results are very sensitive to the size of the sphere and the density of rays that are launched. A better approach is to use ray tubes defined as small bundles of rays with constant flux [29]. The cross-sectional area of a ray tube changes as it propagates and reflects in order to conserve power. For example, a ray tube launched from a point source starts out with a very small cross-sectional area, but it becomes larger as the spherical wavefront spreads out with distance. The fields at the receiver are computed from all the ray tubes that overlap a given receiver point.

The computational burden of ray-shooting is in tracking a very large number of rays through many reflections. A dense enough grid of rays must be launched initially such that the separation between adjacent rays is not more than about half a wavelength at the receiver. To reduce the number of rays that must be tracked, the rays may be launched using a much coarser grid initially to find which rays reach the vicinity of the receiver, and then are adaptively subdivided.

Diffraction is a major problem in ray-shooting approaches because each diffraction from an edge gives rise to a new fan of rays launched from that point, as seen in Figure 19(a). Some of these diffracted rays may hit another edge, and thus the problem compounds quickly. For this reason the order of diffractions is usually limited to only one or two in ray-shooting codes. This may cause some important mechanisms to be left out. 


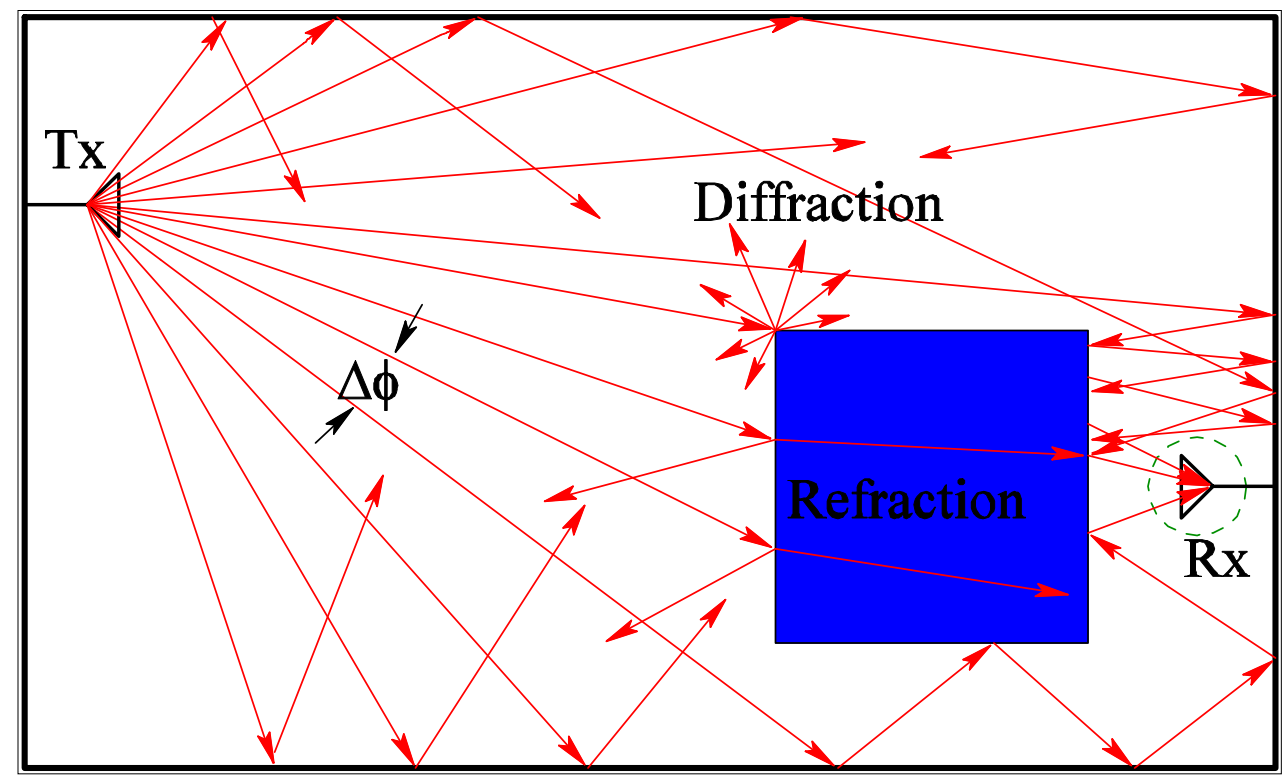

(a) Ray-shooting.

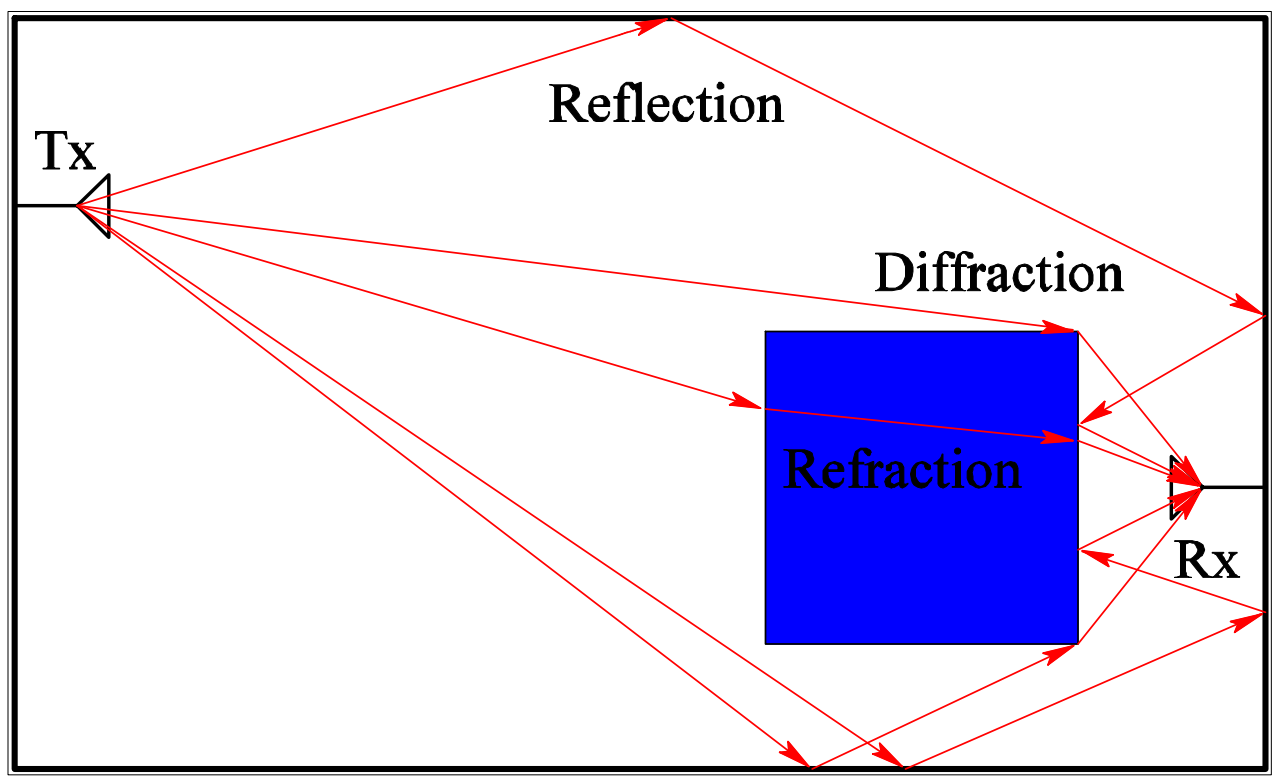

(b) Ray-tracing.

Figure 19: Ray tracking methods for propagation modeling. 
Ray-shooting methods are very versatile and can handle arbitrarily complex geometries, as long as the reflection and transmission properties of the materials are known. They are very useful for indoor and urban propagation problems with a high degree of multipath (multi-bounce). They are not as useful for propagation over hilly terrain or over rooftops because these scenarios tend to be dominated by high-order diffraction effects rather than reflections and refractions. Rayshooting methods are generally not as accurate as numerical methods or ray-tracing methods (discussed next), but are highly efficient for electrically large geometries.

\section{Ray-tracing}

We define here a ray-tracing method as a ray tracking approach where the exact ray paths from source to receiver are found as shown in Figure 3.5(b). Ray-shooting is like hitting a target with a machine gun, whereas ray-tracing is hitting it with good marksmanship. Ray-tracing is sometimes called the image method because image theory may be used to find the reflected ray paths [30].

The ray paths are the actual paths over which EM fields propagate from source to receiver and satisfy the laws of high-frequency asymptotics [31]. Reflected and transmitted rays at material interfaces obey Snell's laws of reflection and refraction. Diffracted rays obey Keller's law of diffraction. In all cases, the ray paths are the shortest distance between the transmitter and receiver for any given combination of reflections, refractions and diffractions. The uniform theory of diffraction (UTD) uses ray-tracing to find the ray paths by which the geometrical optics and diffracted rays reach the receiver [31].

Ray-tracing is potentially very accurate if all the dominant ray paths can be found. However, this may not be easy for complex geometries. First order paths, such as single reflections and diffractions, are fairly easy to find. However, higher-order paths involving multiple reflections and/or diffractions are much more difficult and the search time compounds quickly. For this reason the number of reflections and diffractions are limited in a general ray-tracing code.

Ray-tracing methods are not as good as ray-shooting methods for scenarios with a high degree of multipath. They are most useful for propagation problems with low complexity where high accuracy is needed, and the problem is too large for numerical analysis. Ray-tracing methods have been successfully applied to many outdoor environments, such as propagation over hilly terrain and rooftops [32], and around corners of buildings, as illustrated in Figure 20. In general, ray-tracing methods are better for diffraction-dominant propagation, whereas ray-shooting is better for reflection-dominant propagation. 


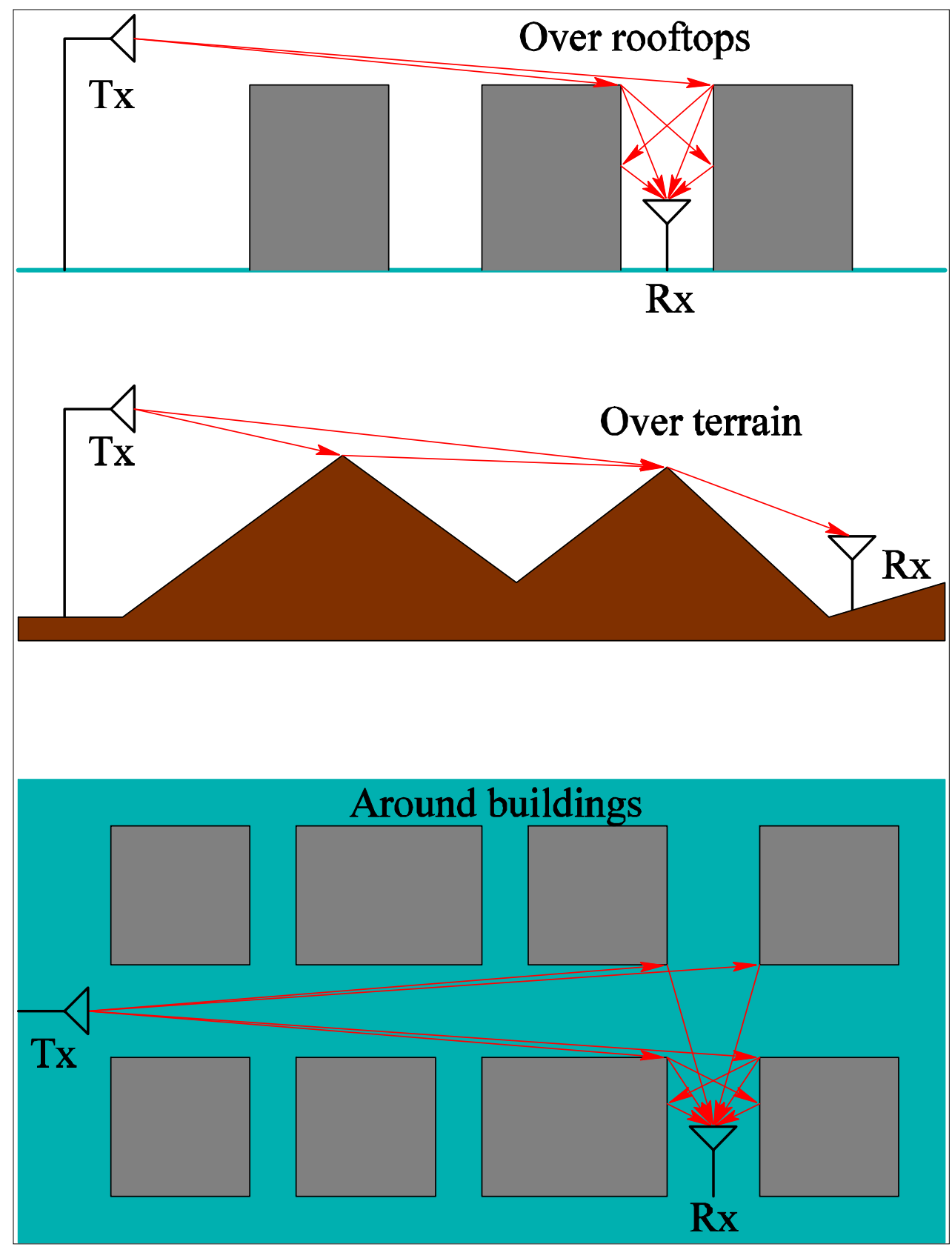

Figure 20: Outdoor propagation problems using ray-tracing. 


\section{Antenna pattern effects}

Rays launched and received by antennas are weighted by the far-field pattern, or radiation pattern. The electric field of a ray launched into free space from a transmitting antenna has the following form [12],

$$
\bar{E}(r, \theta, \phi)=V_{i n} \bar{F}(\theta, \phi) \frac{e^{-j k r}}{r}
$$

where $(r, \theta, \phi)$ are the spherical coordinates of the antenna and $V_{\text {in }}$ is the input voltage at the terminals. $\bar{F}(\theta, \phi)$ is the pattern function which may in general be complex and have $\hat{\theta}$ and $\hat{\phi}$ components. (The electric and magnetic fields of a ray are always transverse to the ray direction $\hat{r}$.) The form of (3.17) is only valid if the observer is in the far field of the antenna, which is defined to be $2 D^{2} / \lambda$ where $D$ is the maximum physical dimension of the antenna. It is noted that the pattern function as written in (3.17) includes the input power, impedance and efficiency of the antenna, and is a function of frequency.

For the receiving case, the effective height $\bar{h}$ determines the received signal at the terminals of the antenna. It is defined by,

$$
V_{\text {out }}=\bar{h}(\theta, \phi) \cdot \bar{E}^{i}
$$

where $V_{\text {out }}$ is the open-circuit output voltage at the terminals and $\bar{E}^{i}$ is the locally planar electric field incident on the antenna. Again, this expression is only valid if the source of the incident field is in the far field of the receiving antenna. It can be shown that the effective height is directly related to the radiation pattern, and includes the efficiency.

\subsubsection{Hybrid Methods}

Hybrid methods are a combination of two or more modeling methods to handle problems better than a single approach, usually for reasons of efficiency. For example, numerical methods are often used to model antennas, and then combined with ray methods to model wide-area propagation environments. Numerical methods may also be used to obtain ray reflection and transmission coefficients for complex inhomogeneous materials such as walls and floors in buildings.

The key to hybrid methods is to properly define the interface or hand-off between two different analysis procedures. This may be done with a simple first order approximation or selfconsistently. Self-consistent approaches systematically include all multiple interactions between the two problem domains. Depending on the problem, the interactions higher than first order may often be neglected. In fact, hybrid methods are usually set up so that the higher order interactions are weak. Here we will describe a typical first order hybrid procedure. 
First order hybrid approaches are done in a decoupled sense. Figure 21 illustrates an example. First, the transmitter (Tx) domain is analyzed in free space using a numerical method. This analysis provides the radiation pattern $\bar{F}(\theta, \phi)$ which is then used in (3.17) with a ray method for the propagation domain. Here we assume the obstacles and the receiver $(\mathrm{Rx})$ domain are in the far field of the transmitter. The rays are tracked from the surface $S_{T}$ to a surface $S_{R}$ enclosing the receiver domain. Lastly, the incident fields on $S_{R}$ are used as the excitation for the numerical solution of the Rx domain. Since the receiver is mounted inside a building, we might not be able to include the building as part of the propagation domain, so we have included it as part of the Rx domain. In this case the incident fields must be found over the entire surface $S_{R}$.

The finite element-boundary integral (FEBI) method [33] is natural for the problem geometry of Figure 21. It is a combination of FEM and MoM where the outer FEM boundary is coupled to an integral equation. The boundary integral is over SR and the FEM region is the building containing the receiver. The incident ray field provides the excitation for the boundary integral.

The above example is typical of an outdoor-to-indoor propagation problem. The obstacle in the propagation domain could also be contained in its own domain and analyzed with a numerical method if it is very complex, as could the Tx antenna if it is a large array mounted on a complex platform, for example. In this latter case the simple far field form of (3.17) could not be used. Instead, we would be required to find an equivalent set of sources which would radiate the same fields in free space as the original antenna. A simple solution is to replace each of the smaller antennas in the array with a source of the form represented by (3.17), but these sources would include the effects of mutual coupling between antenna elements and the platform.

Hybrid methods are useful for realistic scenarios because they can adapt two or more methods to cover a wider range of modeling needs. However, they are much more difficult to implement because the different solutions need to be properly interfaced. This becomes even more complicated if one attempts to combine two different software packages. It may be better to start with a good code based on a single method, such as FEBI, and upgrade it by implementing another EM modeling method such as ray tracing. This way the same geometry representation can be used for both methods. 


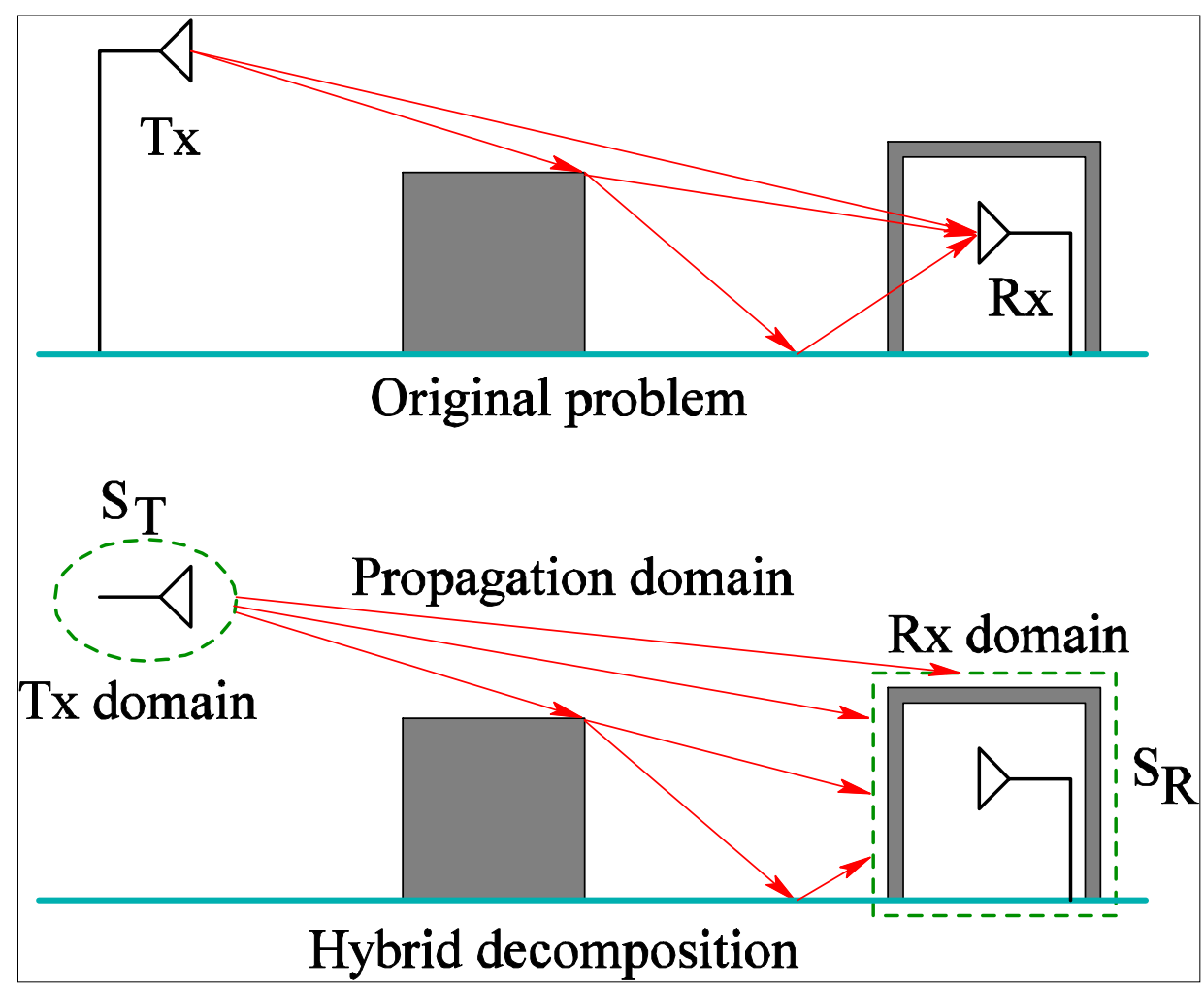

Figure 21: Domain decomposition of a wireless propagation problem for hybrid analysis. 


\subsubsection{Summary of Modeling Methods and Applications}

In this section we will summarize the EM modeling methods described earlier in terms of advantages, limitations, and applications.

Finite-Difference Time Domain

\section{Advantages}

- High accuracy matrix-free numerical solution

- Good for modeling inhomogeneous material structures

- Good for wideband problems

Disadvantages

- Stairstep approximation of geometry

- Requires volumetric mesh

- Requires structured mesh, finest detail or highest dielectric/magnetic material determines cell size for the entire mesh

- Limited to 3D problems smaller than about 10 wavelengths maximum linear dimension

Finite Element Method

\begin{tabular}{|ll|}
\hline \multicolumn{1}{|c|}{ Advantages } \\
\hline - & High accuracy sparse-matrix numerical solution \\
- & Good for modeling inhomogeneous material structures \\
\hline & \multicolumn{1}{c|}{ Disadvantages } \\
\hline - & Requires volumetric mesh \\
- & Dispersion error requires a denser mesh for larger problems \\
- & Larrowband - solution needs to be repeated for each new frequency \\
& dimited to 3D problems smaller than about 10 wavelengths maximum linear \\
\hline
\end{tabular}

Method of Moments

\begin{tabular}{|l|}
\hline \multicolumn{1}{|c|}{ Advantages } \\
\hline - High accuracy numerical solution \\
- Surfaces of geometry are meshed instead of volume \\
- Good for problems with large regions of homogeneous material (such as free space) \\
- Accelerated versions have been developed \\
\hline - \\
- Dives rise to dense system matrix \\
- Limited to 3D problems smaller than about 30 wavelengths maximum linear \\
\end{tabular}




\begin{tabular}{|l|}
\hline \multicolumn{1}{|c|}{ Advantages } \\
\hline - Highly efficient - solution time is relatively independent of frequency \\
- High-order multibounce is included \\
- diffraction \\
- Good for propagation in outdoor urban environments and inside buildings \\
\hline - - Nood for wideband problems \\
\hline - Requires a very large number of rays to be tracked \\
- Neglect of high-order diffraction effects may cause regions of zero signal \\
- Not good for propagation over rooftops or hilly terrain
\end{tabular}

Ray Tracing

\begin{tabular}{|l|}
\hline \multicolumn{1}{|c|}{ Advantages } \\
\hline - Highly efficient - solution time is completely independent of frequency \\
- High-order diffraction may be included \\
- multibounce \\
- Good for propagation over rooftops and hilly terrain, and around isolated buildings \\
\hline - \\
\hline - Not as accurate as numerical methods \\
- Requires a search for all dominant ray paths \\
- Noglect of high-order multibounce may miss important multipath signals \\
\hline
\end{tabular}

\section{Hybrid Methods}

Hybrid Methods
\begin{tabular}{|ll|}
\hline \multicolumn{1}{c|}{ Advantages } \\
\hline - Realistically complex scenarios may be modeled with the best method for each region \\
of the problem
\end{tabular}
- Regions can be analyzed sequentially rather than simultaneously, allowing large
problems to be decoupled.




\subsection{Recommended Approaches to Site-Specific Modeling}

In view of the communications channels of interest that may be obtained by EM modeling, and methods available, the following are a set of modeling recipes for the basic site-specific classes of propagation environments. We assume frequencies of $800 \mathrm{MHz}$ and higher. The recipes may be used for intentional or unintentional (interference) signals. Note that the reverse propagation from that listed may be found via reciprocity.

\subsubsection{Outdoor Propagation}

Propagation mechanisms: Reflection, diffraction, and diffuse scattering from terrain, buildings, vehicles, etc.

Modeling approach:

1. Analyze transmitting antenna and platform using a numerical method such as MoM, FEM, or FDTD. Obtain a set of equivalent directive sources with known radiation patterns.

2. Track the fields from the transmitter to the receiver using ray shooting or ray tracing, depending on the type of outdoor environment.

3. Analyze the receiving antenna and platform with a numerical method, using the incident ray fields as excitation. Compute the received signal at the terminals of the antenna.

\subsubsection{Outdoor-to-Indoor Propagation, Large Room}

Propagation mechanisms: Same as outdoor, plus transmission through walls, floors, ceilings, windows, doors, etc.

Modeling approach: Same as outdoor, but add transmission of ray fields into the room where receiver is located. Include diffraction from windows and doors. Numerical methods may be used to compute ray transmission coefficients of inhomogeneous walls, floors and ceilings.

\subsubsection{Outdoor-to-Indoor Propagation, Small Room or Enclosure}

Propagation mechanisms: Same as outdoor, plus complex transmission into room or enclosure where receiver is located, and multipath inside the room. 


\section{Modeling approach:}

1. Analyze transmitting antenna and platform using a numerical method such as MoM, FEM, or FDTD. Obtain a set of equivalent directive sources with known radiation patterns.

2. Track the fields from the transmitter to the outer boundary of the room using ray shooting or ray tracing.

3. Use a numerical method (FEM, FDTD, MoM) to analyze the room and all materials, with the incident ray fields on the outer boundary as excitation. The FEBI method is wellsuited for this task. Compute the fields incident on the receiving antenna.

4. Analyze the receiving antenna and platform with a numerical method, using the fields from step 3 as excitation. Compute the received signal at the terminals of the antenna. If the enclosure is very small it may be necessary to combine this step with step 3 .

\subsubsection{Indoor Propagation, Large Room or Buiolding}

Propagation mechanisms: Reflection, diffraction, and transmission associated with walls, floors, ceilings, doorways, furniture, etc.

Modeling approach:

1. Analyze transmitting antenna and platform using a numerical method such as MoM, FEM, or FDTD. Obtain a set of equivalent directive sources with known radiation patterns.

2. Track the fields from the transmitter to the receiver using ray shooting. Numerical methods may be used to compute ray transmission coefficients of inhomogeneous walls, floors and ceilings. A hybrid method may be used to model a complex obstacle in the propagation path.

3. Analyze the receiving antenna and platform with a numerical method, using the incident ray fields as excitation. Compute the received signal at the terminals of the antenna.

\subsubsection{Indoor Propagation, Small Room or Enclosure}

Propagation mechanisms: Complex multipath involving inhomogeneous materials and obstacles.

Modeling approach:

1. Analyze transmitting antenna and platform using a numerical method such as MoM, FEM, or FDTD. Obtain a set of equivalent directive sources with known radiation patterns. 
2. Use a numerical method (FEM, FDTD, MoM) to analyze the room and all materials, with the sources from step 1 as excitation. Compute the fields incident on the receiving antenna.

3. Analyze the receiving antenna and platform with a numerical method, using the fields from step 2 as excitation. Compute the received signal at the terminals of the antenna. If the enclosure is very small it may be necessary to combine this step with step 2 .

Note: It may be necessary to combine all three steps into one numerical analysis of the entire system. If the enclosure is highly reverberant, there may be strong multiple interactions between the antennas and the environment.

\subsection{Accuracy and Modeling Fidelity}

Given that communications channels are characterized statistically, the question arises, how much accuracy and detail do we need in our EM modeling solutions? If we model a given sitespecific scenario in great detail, the slightest change or error in the geometry could greatly affect the EM fields, but most of the statistics probably would not change. The answer to this question depends on which of the channel parameters defined in Section 2.4.1 we are computing.

- The mean received signal power (MRSP) describes the average signal energy reaching the receiver, where averaging is performed globally over time and frequency, and locally over location. As seen in Figure 12, it is affected by path loss, shadowing (large-scale fading) and multipath (small-scale fading). Path loss is just the average slope of the MRSP curve as a function of distance from the transmitter, and depends on macro features such as terrain, buildings, walls, floors, etc. Modeling fidelity is therefore not critical for path loss, as long as the large features of the environment are included.

- The small-scale fading pattern associated with multipath is an interference phenomenon, and it is very difficult to predict the peaks and nulls exactly. However, it is not necessary to know the exact fading pattern as long as we can predict the type of fading (e.g., Rayleigh or Ricean) and the local mean and standard deviation of the signal. Again, modeling fidelity is not critical as long as we include enough of the scattering features responsible for the multipath.

- $\quad$ Large-scale fading due to shadowing (e.g., blockage or attenuation) is the most important to model correctly. It determines if a signal can penetrate into a building, for example, and through walls, floors, doorways, and windows. Other examples include diffraction around buildings and terrain features. On a smaller scale it might include shadowing by objects in a room, such as furniture or a person walking between the transmitter and receiver. A good EM model must consider these situations and produce a large number of statistical samples to reflect the variability of the environment.

The accurate modeling of large-scale fading is especially critical to predicting the penetration of interfering signals into shielded communications systems. For example, external signals could enter a shielded room through a crack under the door, through 
wiring conduits, or even directly through the shielding if the signal is strong enough. These situations require a detailed numerical model with very accurate material parameters.

- The spatial covariance quantifies how quickly the MRSP changes as a function of position. Again, this is a function of small-scale fading due to multipath. As mentioned above, EM modeling accuracy is not critical because we do not need to predict the exact locations of peaks and nulls of the fading pattern, but only the separation between peaks and nulls. So, we just need to include in the model enough of the scattering features responsible for the multipath.

Spatial covariance is very important to understand in communications systems that take advantage of spatial diversity. Multiple antennas may be used to overcome multipath fading by separating the antennas by the distance between an adjacent peak and null. That way, the antennas should never all be in a null at the same time. Angular diversity is also important to understand because directive antennas may be designed to amplify certain multipath signals and attenuate others in order to mitigate interference and increase channel capacity.

Multiple-input, multiple output (MIMO) systems take advantage of spatial diversity to increase channel capacity with multiple antennas [34]. EM modeling would be very useful for identifying optimal locations for these antennas so that there is low correlation between channels.

- The power delay profile (PDP) is the statistical description of the multipath delay spread, or the distribution of received energy versus time. EM modeling fidelity is not critical because system designers are concerned only with the general shape of the PDP. This will be predicted well by EM modeling as long as enough of the multipath scattering features are included. It should be mentioned that the late-time multipath could be due to very high order reflections and diffractions, so it is important in using ray methods that enough terms are included. Likewise, large scattering obstacles that are very far away from the transmitter and receiver could cause a significant late-time multipath signal.

For a given receiver location, the PDP is made up of many shifted impulses in time. Since the bandwidth is not infinite, these impulses will be broadened according to the bandwidth of the system. Therefore, the modeling precision does not need to be significantly greater than that required by the resolution of these impulses. For example, an impulse with $200 \mathrm{MHz}$ bandwidth has a pulse width of about $5 \mathrm{~ns}$, which corresponds to $1.5 \mathrm{~m}$ resolution. This is much larger than the wavelength at gigahertz frequencies. This does not mean that numerical methods may use a very coarse mesh (although the tenth of a wavelength meshing criterion might be relaxed some). It means that objects in the propagation environment do not need to be precisely located.

- The Doppler power profile is the statistical description of the channel's time variation. This is very important for mobile communications, as well as in urban settings and office buildings where there could be a large number of moving objects contributing to multipath. EM modeling may be used to predict Doppler effects by stepping the 
geometry and solving the EM problem at discrete moments in time [35, 36]. An object's velocity determines how much it moves at each time step. Modeling fidelity may be important here to distinguish the movement, depending on the time step and velocity.

It is clear from the above discussion that accuracy and fidelity of the EM model is not critical for most of the channel parameters of interest, with the exception of blockage and attenuation. There does not appear to be much in the literature about the effect of modeling fidelity in wireless applications, and is a promising topic for future research. It may be possible to extend the range of numerical methods considerably by relaxing the constraints on mesh density. An example is the iterative physical optics (IPO) method [37] which extends the MoM to higher frequencies by incorporating some high-frequency approximations and reducing the mesh density from 10 points per wavelength to only 2 or 3 points per wavelength. The asymptotic phasefront extraction (APEx) method also extends the MoM to higher frequencies by incorporating electrically large traveling wave basis functions. 
Intentionally Left Blank 


\section{Research Directions}

In this report we have summarized the current state-of-the-art in analyzing communications channels with computer simulations. Some areas for potential research and development have become apparent.

One area is to investigate the effect of CEM numerical accuracy and geometry fidelity on the channel parameters to be computed. It was shown in Section 3.5 that most of the parameters are relatively insensitive to modeling fidelity, with the exception of some special cases where signal penetration must be accurately predicted. However, it is not known to what degree the precision of the geometric model and the accuracy of the CEM solution may be relaxed. For example, do we need 10 sample points per wavelength for numerical methods? Do we need to model buildings and rooms with a precision of $1 \mathrm{~cm}$ when the system bandwidth only requires $1.5 \mathrm{~m}$ resolution?

A related area of research is the application of new coarse-grid techniques such as iterative physical optics [37] and asymptotic phasefront extraction [38]. These methods extend the range of applicability of standard numerical methods and incorporate more of the propagation physics than ray methods. It is possible they could replace ray methods for some of the smaller problems, such as indoor propagation and maybe even urban propagation.

Another area of research that has not been discussed much is the propagation of wireless signals through the ground and in underground structures. This area needs to be developed to understand how signals penetrate into underground structures and to evaluate shielding effectiveness. 
Intentionally Left Blank 


\section{References}

[1] D. Tse and P. Viswanath, Fundamentals of Wireless Communication. New York: Cambridge University Press, 2005.

[2] A. Papoulis, Probability, Random Variables, and Stochastic Processes. New York: McGraw-Hill, 3rd ed., 1991.

[3] B. Sklar, Digital Communications: Fundamentals and Applications. Englewood Cliffs, TX: Prentice Hall, 2nd ed., 2001.

[4] H. Dai and H. V. Poor, "Advanced signal processing for power line communications," IEEE Communications Magazine, vol. 41, pp. $100\{107$, May 2003.

[5] S. Verdu, Multiuser Detection. New York: Cambridge, 1998.

[6] C. E. Shannon, "A mathematical theory of communication,” Bell System Technical Journal, vol. 27, pp. 379-423 and 623-656, 1948.

[7] W. C. Jakes, Microwave Mobile Communications. Wiley, 1974. [reprinted by IEEE Press].

[8] D. Parsons, The Mobile Radio Propagation Channel. New York: Wiley, 1994.

[9] T. S. Rappaport, Wireless Communications: Principles and Practice. Englewood Cliffs, NJ: Prentice-Hall, 2nd ed., 2001.

[10] M. Patzold, Mobile Fading Channels. New York: Wiley, 2002.

[11] A. Goldsmith, Wireless Communications. New York: Cambridge University Press, 2005.

[12] W. L. Stutzman and G. A. Thiele, Antenna Theory and Design, John Wiley \& Sons, New York, 1981.

[13] C. A. Balanis, Antenna Theory Analysis and Design, Harper \& Row, New York, 1982.

[14] Antenna Engineering Handbook, R. C. Johnson and H. Jasik editors, McGraw-Hill Book Company, New York, 1984.

[15] A. F. Peterson, S. L. Ray and R. Mittra, Computational Methods for Electromagnetics, IEEE PRESS Series on Electromagnetic Waves, IEEE Press, New York, 1998.

[16] R. W. Kindt and J. L. Volakis, “Array decomposition-fast multipole method for finite array analysis," Radio Science, 39(2), Art. No. RS2018, April 16, 2004. 
[17] R. F. Harrington, Time-Harmonic Electromagnetic Fields, McGraw-Hill Book Company, New York, 1961.

[18] T. K. Sarkar, Z. Ji, K. Kim, A. Medouri and M. Salazar-Palma, "A survey of various propagation models for mobile communication," IEEE Antennas and Propagation Mag., 45 (3):51-82, June 2003.

[19] K. S. Kunz and R. J. Luebbers, The Finite Difference Time Domain Method for Electromagnetics, CRC Press, Boca Raton, FL, 1993.

[20] K. S. Yee, "Numerical solution of initial boundary value problems involving Maxwell's equations in isotropic media," IEEE Trans. on Antennas and Propagation, 14(5):302207, May 1966.

[21] J. P. Berenger, "Perfectly matched layer for the FDTD solution of wave-structure interaction problems," IEEE Trans. on Antennas and Propagation, 44(1):110-117, Jan. 1996.

[22] R. Holland, V. P. Cable and L. C. Wilson, "Finite-volume time-domain (FVTD) techniques for EM scattering," IEEE Trans. on Electromagnetic Compatibility, 33(4):281-294, Nov. 1991.

[23] P. P. Silvester and R. L. Ferrari, Finite Elements for Electrical Engineers, Cambridge University Press, 1983.

[24] R. F. Harrington, Field Computation by Moment Methods, Macmillan, New York, 1968.

[25] S. M. Rao, D. R. Wilton and A. W. Glisson, "Electromagnetic scattering by surfaces of arbitrary shape," IEEE Trans. on Antennas and Propagation, 30(5):409-418, May 1982.

[26] R. Coifman, V. Rokhlin and S. Wandzura, "The fast multipole method for the wave equation: a pedestrian prescription," IEEE Antennas and Propagation Mag., 35(3):712,June 1993.

[27] S. H. Chen and S. K. Jeng, "SBR/image approach for radio wave propagation in indoor environments with metallic furniture," IEEE Trans. on Antennas and Propagation, 45(1):98-106, Jan. 1997.

[28] W. Lu and K. T. Chan, "Advanced 3D ray tracing method for indoor propagation prediction," Electronics Letters, 34(12):1259-1260, June 11, 1998.

[29] C. F. Yang, B. C. Wu and C. J. Ko, "A ray-tracing method for modeling indoor wave propagation and penetration," IEEE Trans. on Antennas and Propagation, 46(6):907919, June 1998.

[30] J. W. Mckown and R. L. Hamilton, Jr., "Ray tracing as a design tool for radio networks," IEEE Network Mag., 5:27-30, Nov. 1991. 
[31] P. H. Pathak, "Techniques for high frequency problems," in Antenna Handbook, Theory, Application and Design, Y.T. Lo and S.W. Lee, Eds., Van Nostrand Reinhold, New York, 1988.

[32] R. J. Luebbers, "Finite conductivity uniform GTD versus knife edge diffraction in prediction of propagation path loss," IEEE Trans. on Antennas and Propagation, 32(1):70-76, Jan. 1984.

[33] M. A. Morgan, C. H. Chen, S. C. Hill and P. W. Barber, "Finite element-boundary integral formulation for electromagnetic scattering," Wave Motion, 6(1):91-103, Jan. 1984.

[34] M. A. Jensen and J. W. Wallace, "A review of antennas and propagation for MIMO wireless communication," IEEE Trans. on Antennas and Propagation, 52(11), Nov. 2004.

[35] J. Van Bladel, "Electromagnetic fields in the presence of rotating bodies," Proc. IEEE, 64:301-318, March 1976.

[36] R. D. Graglia, A. Freni and G. Pelosi, "A finite element approach to the electromagnetic interaction with rotating penetrable cylinders of arbitrary cross section," IEEE Trans. on Antennas and Propagation, 41(5):635-650, May 1993.

[37] F. Obelleiro, J. L. Rodriguez and R. J. Burkholder, “An Iterative Physical Optics Approach for Analyzing the Electromagnetic Scattering by Large Open-Ended Cavities," IEEE Transactions on Antennas and Propagation, 43(4):356-361, April 1995.

[38] D. H. Kwon, R. J. Burkholder and P. H. Pathak, 'Efficient Method of Moments (MoM) Formulation for Large PEC Electromagnetic Scattering Problems using Asymptotic Phasefront Extraction," IEEE Trans. on Antennas and Propagation, 49(4):583-591, April 2001. 


\section{Distribution}

$\begin{array}{lll}1 & \text { MS 1152 } & \text { Mark Kiefer, 1652 } \\ 1 & \text { MS 9035 } & \text { Bill even, 8220 } \\ 1 & \text { MS 9153 } & \text { Greg Thomas, 8240 } \\ 1 & \text { MS 9108 } & \text { Curt Nilsen, 8229 } \\ 1 & \text { MS 9106 } & \text { Ed Talbot, 8226 } \\ 1 & \text { MS 9154 } & \text { Phil Bryson, 8244 } \\ 1 & \text { MS 9108 } & \text { Chrisma Jackson, 8229 } \\ 1 & \text { MS 9154 } & \text { Cheryl Lam, 8244 } \\ 1 & \text { MS 9202 } & \text { Brian Owens, 8205 } \\ 1 & \text { MS 9102 } & \text { Ratish Punnoose, 8235 } \\ 1 & \text { MS 9404 } & \text { Bob Carling, 8700 } \\ 1 & \text { MS 9404 } & \text { Davina Kwon, 8770 } \\ 1 & \text { MS 9042 } & \text { Mike Chiesa, Manager, 8774 } \\ 1 & \text { MS 9042 } & \text { Michael Forman, 8774 } \\ 4 & \text { MS 9042 } & \text { Robert Mariano, 8774 } \\ 1 & \text { MS 9011 } & \text { John Howard, 8941 } \\ & & \\ 1 & \text { MS 0899 } & \text { Technical Library, 9616 } \\ 2 & \text { MS 9018 } & \text { Central Technical Files, 8944 }\end{array}$

